This item was submitted to Loughborough's Research Repository by the author.

Items in Figshare are protected by copyright, with all rights reserved, unless otherwise indicated.

\title{
An assessment of large eddy simulations of premixed flames propagating past repeated obstacles
}

PLEASE CITE THE PUBLISHED VERSION

PUBLISHER

(c) Taylor \& Francis

VERSION

AM (Accepted Manuscript)

LICENCE

CC BY-NC-ND 4.0

\section{REPOSITORY RECORD}

Gubba, Sreenivasa Rao, Salah S. Ibrahim, W. Malalasekera, and Assaad R. Masri. 2019. "An Assessment of Large Eddy Simulations of Premixed Flames Propagating Past Repeated Obstacles”. figshare. https://hdl.handle.net/2134/5497. 
This item was submitted to Loughborough's Institutional Repository (https://dspace.lboro.ac.uk/) by the author and is made available under the following Creative Commons Licence conditions.

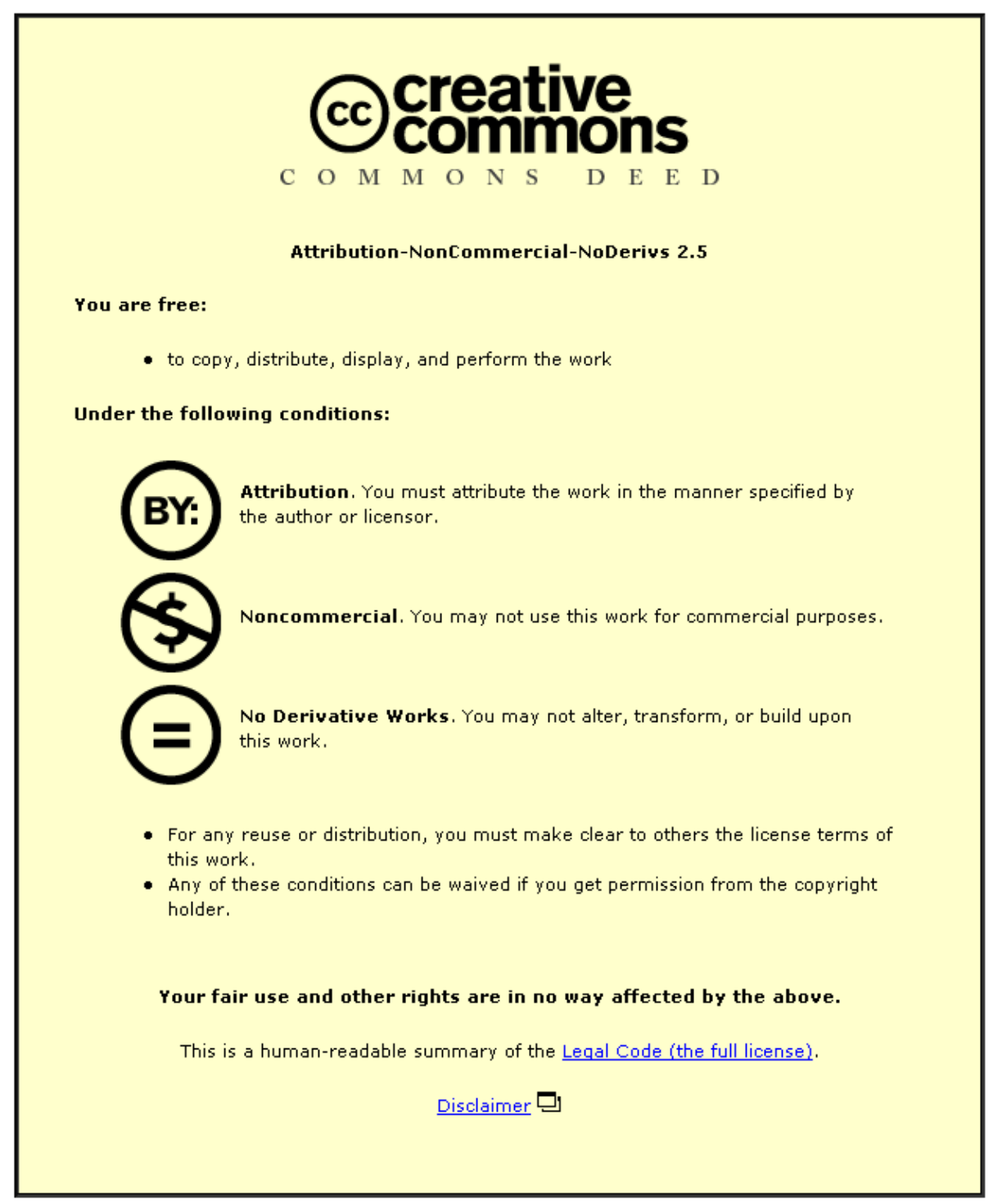

For the full text of this licence, please go to: http://creativecommons.org/licenses/by-nc-nd/2.5/ 
Submitted to Combustion Theory and Modelling, May 2007

Running heads S.R. Gubba et al.

LES of turbulent premixed flame

Article Type

RESEARCH ARTICLE

\section{An Assessment of Large Eddy Simulations of Premixed Flames Propagating past Repeated Obstacles}

\section{Sreenivasa Rao Gubba ${ }^{a}$, Salah S Ibrahim*, ${ }^{*}$, Weertunge Malalasekera ${ }^{\mathrm{c}}$ and Assaad R Masri ${ }^{\mathbf{d}}$}

${ }^{\text {a }}$ Wolfson school of Mechanical and Manufacturing Engineering Loughborough University Loughborough, LE11 3TU UK

S.R.Gubba@lboro.ac.uk

*, b Aeronautical and Automotive Engineering

Loughborough University Loughborough LE11 3TU UK

Phone: +44 1509 227253, Fax: +44 1509227275

S.S.Ibrahim@lboro.ac.uk

${ }^{\mathrm{c}}$ Wolfson school of Mechanical and Manufacturing Engineering Loughborough University Loughborough, LE11 3TU UK W.Malalsekera@lboro.ac.uk

${ }^{\mathrm{d}}$ School of Aerospace, Mechanical and Mechatronic Engineering The University of Sydney, NSW, 2006, Australia masri@aeromech.usyd.edu.au

*Corresponding author

Word length of this paper: Text

Equations 22

References $\quad 740$

Figures $\quad 600$

Total $\mathbf{9 8 8 4}$ 


\begin{abstract}
This paper presents an assessment of Large Eddy Simulations in calculating the structure of turbulent premixed flames propagating past solid obstacles. One objective of the present study is to evaluate the LES simulations and identify the drawbacks in accounting the chemical reaction rate. Another objective is to analyse the flame structure and to calculate flame speed, generated overpressure at different time intervals following ignition of a stoichiometric propane/air mixture. The combustion chamber has built-in repeated solid obstructions to enhance the turbulence level and hence increase the flame propagating speed. Various numerical tests have also been carried out to determine the regimes of combustion at different stages of the flame propagation. These have been identified from the calculated results for the flow and flame characteristic parameters. It is found that the flame lies within the 'thin reaction zone' regime which supports the use of the laminar flamelet approach for modelling turbulent premixed flames. A sub-model to calculate the model coefficient in the algebraic flame surface density model is implemented and examined. It is found that the LES predictions are slightly improved due to the calculation of model coefficient by using sub-model. Results are presented and discussed in this paper are for the flame structure, position, speed, generated pressure and the regimes of combustion during all stages of flame propagation from ignition to venting. The calculated results are validated against available experimental data.
\end{abstract}

Keywords: Turbulent premixed flames, LES, Turbulence, Regimes of combustion, Flame surface density 


\section{Introduction}

Large Eddy Simulations (LES) are now accepted as a feasible computational tool in turbulent combustion [1-5] despite the added computational cost and the need for subgrid scale (SGS) modeling. LES has a clear advantage over classical Reynolds averaged methods in the capability of accounting of time-varying nature of the flows and this is particularly important in transient processes such as swirling flows or turbulent premixed propagating flames. The ever increasing speed of computers is rendering the high computational requirement of LES quite feasible and shifting the focus towards developing adequate SGS models to enable these approaches to account effectively for combustion. While such a need is prevalent in both premixed and non-premixed flames, the focus of this paper is on the former. Premixed combustion is encountered in many engineering applications such as spark ignition engines, gas turbines and accidental explosion events. Outstanding research issues associated with the structure of the flame front and the regimes of combustion as the flame front propagates through highly turbulent flow field are further complicated by instabilities, which occur due to the unsteady nature of the flow. Understanding such issues is central to the development of advanced physical sub-models that improve current predictive capabilities for turbulent premixed flames.

Inspired by the experimental work of Masri et al [1\&6], the present investigation is carried out using LES technique to analyse the turbulent flame structure and to identify the regimes of combustion of a turbulent premixed propagating flame in a confined chamber having solid obstructions. The laboratory scale premixed combustion chamber established by The University of Sydney combustion group [1\&6] is considered here to validate the LES predictions. In these experiments, a stagnant, stoichiometric propane/air mixture is ignited and a propagating turbulent flame is generated. The chamber has several built-in solid obstructions to enhance the turbulence levels thereby increasing the flame propagating speed, leading to strong interactions between the flame, flow and the solid obstructions. 
In LES, large eddies above a cut-off length scale are resolved and the small ones are modelled employing SGS models. Several modelling approaches such as flame surface density (FSD) [7\&8], flame tracking technique (G-equation) [9-11], artificially thickened modelling [12] and probability density function (PDF) [13] are successfully adapted from Reynolds Averaged Navier-Stokes (RANS) to LES. The FSD models are well established in accounting for the chemical reactions in the context of LES [14\&15], where the rate of combustion is expressed as a function of a reaction progress variable and the filter width. The FSD modelling approach [14] is considered in the present study and efforts are made to calculate the model coefficient by employing a sub-model.

Another essential requirement for the maturity of LES as a reliable numerical tool is, the need to establish methodologies for obtaining solutions that are independent of the size of the grid resolution and filter width. Currently, most formulations link the filter size to the numerical grid and these are referred to as implicit methods. The sub-grid filter must also be sufficiently fine to resolve a significant proportion of the turbulent kinetic energy [16]. This paper attempts to shed more light on the issue of obtaining grid independent LES and investigates this issue with respect to both the gird size and the filter width.

Combustion models are derived based on the physical analysis and comparisons of the various time and length scales involved in combustion phenomena. These comparisons lead to the 'turbulent combustion diagrams' where various regimes are recognized and delineated by introducing dimensionless characteristic numbers. These diagrams indicate whether the flow contains flamelets, pockets or distributed reaction zones depending on turbulence characteristics (integral length scales, turbulent kinetic energy etc). In general, and for premixed turbulent propagating flames, various regimes of combustion may be present depending on the level and scale of the local flow turbulence. These regimes have been the subject of active research over the past few years [7, 9, 17\&18]. Identifying the regimes of turbulent premixed flames in combustion systems can help out in developing a new model and can examine existing models. 
Turbulent combustion regimes are generally represented on the basis of two dimensionless numbers, namely, Damköhler number (Da) [19] and Karlovitz number (Ka). Three regimes can be identified based on these numbers as corrugated flamelets, thin reaction zones, and broken reaction zones. The majority of the combustion devices are operated in the thin reaction zones regime, because mixing is superior at higher Ka numbers, which leads to higher volumetric heat release and shorter combustion times [20]. Thin reaction zone regime, is identified where the Kolmogorov scale becomes smaller than the flame thickness, which implies $\mathrm{Ka}>1$. In the corrugated flamelet regime, the laminar flame thickness is smaller than the Kolmogorov length scale, and hence $\mathrm{Ka}<1$.

The work presented in this paper is organized as follows: Section 2 describes the details of the combustion chamber used for validation and highlighted the importance of placing solid obstacles inside the chamber. Section 3 presents the governing equations and the mathematical procedure used. Section 4 explains the numerical procedure used in LES calculations. Section 5 presents the results and discussion of the present LES simulations. The summery of the conclusions are presented in Section 6.

\section{The Test Case}

The test case used here for model validation was that premixed combustion chamber constructed at The University of Sydney [1\&6]. The rig consists of a laboratory scale premixed combustion chamber with built-in repeated solid obstructions as shown in Figure 1. The published experimental data for the flame structure and generated overpressure are used for model validation. The chamber has a square cross section of $50 \mathrm{~mm}$ and a length of $250 \mathrm{~mm}$. It has a total volume of $0.625 \mathrm{~L}$. Three baffle plates and a square obstacle are placed at different downstream location from the bottom ignition end. Each baffle plate is of 50 x $50 \mathrm{~mm}$ aluminum frame constructed from $3 \mathrm{~mm}$ thick sheet. This consists of five $4 \mathrm{~mm}$ wide bars each with a $5 \mathrm{~mm}$ wide space separating them, rendering a blockage ratio of $40 \%$. The baffle plates are aligned at 90 degrees to the solid obstacle in the configuration employed in the present study. These vented plates are named as S1, S2 and S3 and located at 20, 50 and $80 \mathrm{~mm}$ respectively from the ignition point. A solid square obstacle of $12 \mathrm{~mm}$ in cross section is centrally located 
at $96 \mathrm{~mm}$ from the ignition point running throughout the chamber cross section which causes significant disruption to the flow.

In order to facilitate the discussion in this paper, the chamber has been divided into five regions as shown in Figure 2. The main objective of doing this is to capture and examine the nature of the flow and flame as the flame propagates in the obstructed chamber as discussed in Section 5.3.

\subsection{Arrangement of baffle plates and solid obstacle}

Introduction of baffle plates and obstacles into the flow field inside the combustion chamber serve to increase the turbulence level and hence flame propagation speed. The position and number of the baffle plates employed with respect to the square solid obstacle significantly influence the generated peak pressure, flame speed and structure. From the experimental investigations of Kent et al [6], it is found that the addition of baffle plates increases the overpressure, speeds up the flame and causes significant level of stretching in the flame front as it jets through the baffles. Higher turbulence levels increase the burning rates and hence the overpressures at an even faster rate than the flame propagating speed. Hence, considerably large increase of overpressure is expected due to a small increase in the flame propagating speed. In the present work, the influence of individual baffle plate and the square obstacle on the flow is discussed with particular relevance on how the solid obstructions are arranged inside the chamber to change the turbulence levels and the regimes of combustion.

\section{The Mathematical Model}

Numerical modelling of the turbulent premixed flames in LES is very challenging and usually involved in solving the conservation equations of mass, momentum, energy and a reaction progress variable coupled with the state equation. The work presented in this paper uses the same mathematical model and LES code, PUFFIN described in Masri et al [1]. However brief details of the filtered equations are given for ready reference. 
In order to solve the conservation equations by LES technique, a low pass spatial filter $(F)$ should be applied. The spatial filter $(F)$ can be applied to any flow variable $\phi(x, t)$ to separate large eddies from flow motions, such as:

$$
\bar{\phi}(x, t)=\int_{v} F\left(x-x^{\prime}\right) \phi\left(x^{\prime}, t\right) d x^{\prime}
$$

The integration is carried out over the entire flow domain $v$. A number of spatial filters may be used in LES to resolve the flow field. In the present work, a box filter is used as it fits naturally into the finite volume discretization of the governing equations. The box filter used here is having a characteristic filter width $\bar{\Delta}$ and can be defined as

$$
\bar{\Delta}=(\delta x \delta y \delta z)^{1 / 3}
$$

where, $\delta x, \delta y$ and $\delta z$ are the grid resolutions in three coordinate directions. The Favre filtered (density weighted) transport equations of mass, momentum, energy and reaction progress variable are respectively given by

$$
\begin{gathered}
\frac{\partial \bar{\rho}}{\partial t}+\frac{\partial\left(\bar{\rho} \tilde{u}_{j}\right)}{\partial x_{j}}=0 \\
\frac{\partial\left(\bar{\rho} \tilde{u}_{i}\right)}{\partial t}+\frac{\partial\left(\bar{\rho} \tilde{u}_{i} \tilde{u}_{j}\right)}{\partial x_{j}}=-\frac{\partial \bar{P}}{\partial x_{i}}+\frac{\partial}{\partial x_{j}}\left(2 \bar{\mu}\left[\tilde{S}_{i j}-\frac{1}{3} \delta_{i j} \tilde{S}_{k k}\right]\right)+\frac{\partial \tau_{i j}^{s g s}}{\partial x_{j}} \\
\frac{\partial(\bar{\rho} \tilde{h})}{\partial t}+\frac{\partial\left(\bar{\rho} \tilde{u}_{j} \tilde{h}\right)}{\partial x_{j}}+\frac{\partial\left(\overline{\rho u_{j}^{\prime \prime} h^{\prime \prime}}\right)}{\partial x_{j}}=\frac{\partial \bar{P}}{\partial t}+2 \bar{\mu}\left[\tilde{S}_{i j}-\frac{1}{3} \delta_{i j} \tilde{S}_{k k}\right]: \frac{\partial \tilde{u}_{j}}{\partial x_{i}}+\frac{\partial}{\partial x_{j}}\left(\frac{\bar{\mu}}{\operatorname{Pr}} \frac{\partial \tilde{h}}{\partial x_{j}}\right)+\overline{\dot{q}}_{c} \\
\frac{\partial \bar{\rho} \tilde{c}}{\partial t}+\frac{\partial\left(\bar{\rho} \tilde{u}_{j} \tilde{c}\right)}{\partial x_{j}}+\frac{\partial\left(\overline{\rho u_{j}^{\prime \prime} c^{\prime \prime}}\right)}{\partial x_{j}}=\frac{\partial}{\partial x_{j}}\left(\frac{\bar{\mu}}{\operatorname{Sc}} \frac{\partial \tilde{c}}{\partial x_{j}}\right)+\overline{\dot{\omega}}_{c} \\
\bar{P}=\bar{\rho} R_{G} \tilde{T}
\end{gathered}
$$

In the above equations $\rho$ is the density, $u_{j}$ is the velocity component in $x_{j}$ direction, $P$ is the pressure, $\mu$ is the dynamic viscosity, $S_{i j}$ is stress tensor, $\delta_{i j}$ is Kronoker delta, Pr is Prandtl number, $\dot{q}_{c}$ is chemical source term, $\dot{\omega}_{c}$ is the reaction rate, $c$ is the reaction 
progress variable, which defines the chemical status from zero to one in the unburned mixture and the products respectively, $T$ is the temperature and $R_{G}$ is the gas constant, where $R_{G}=R_{u} / M, R_{u}$ is universal gas constant and $M$ is the molecular mass. Mathematically the reaction progress variable can be derived as, $1-Y_{f u} / Y_{f u}^{0}$. Here $Y_{f u}$ is the local fuel mass fraction and $Y_{f u}^{0}$ is the fuel mass fraction in unburned mixture. An over-bar describes the application of the spatial filter, while the tilde denotes Favre filtered quantities. In order to close the Favre filtered governing equations SGS models for turbulence, scalar flux and chemical reaction rate should be employed. It should be noted here that the buoyancy effects are neglected in the momentum equation. This is mainly due to the higher flame propagation speeds identified from experiments, which supports the observation of small flame residence times within the chamber (typically $13 \mathrm{~ms})$.

\subsection{Modelling of SGS Turbulence}

The filtered NS equation yields an unclosed term, $\tau_{i j}^{s g s}$ due to the decomposition of nonlinear convective terms, which must be closed either by using models or solving complex additional transportation equations. In LES, the term $\tau_{i j}^{s g s}$ is generally referred to as residual stress and represents the impact of the unresolved velocity components on the resolved ones. Mathematically these terms arises from the non-linearity of the convection term, which does not commute with the linear filtering operation. In the present work, this term is modelled by widely used classical Smagorinsky turbulence model [21], based on linear eddy viscosity as:

$$
\begin{array}{r}
\tau_{i j}^{s g s}=\bar{\rho} \overline{u_{i}^{\prime \prime} u_{j}^{\prime \prime}}=\bar{\rho}\left(\widetilde{u_{i} u_{j}}-\tilde{u}_{i} \tilde{u}_{j}\right) \\
\tau_{i j}^{s g s}-\frac{1}{3} \delta_{i j} \tau_{k k}^{s g s}=-2 \bar{\mu}_{\mathrm{SGS}}\left(\tilde{S}_{i j}-\frac{1}{3} \delta_{i j} \tilde{S}_{k k}\right)
\end{array}
$$

where the filtered strain rate tensor $\tilde{S}_{i j}$ is defined as:

$$
\tilde{S}_{i j}=\frac{1}{2}\left(\frac{\partial \tilde{u}_{i}}{\partial x_{j}}+\frac{\partial \tilde{u}_{j}}{\partial x_{i}}\right)
$$


In equation (9), the SGS eddy viscosity $\bar{\mu}_{\mathrm{SGS}}$ a function of the filter size and the strain rate,

$$
\bar{\mu}_{\mathrm{SGS}}=\bar{\rho}\left(C_{s} \bar{\Delta}\right)^{2}|\tilde{S}|
$$

where $|\tilde{S}|=\sqrt{2 \tilde{S}_{i j} \tilde{S}_{i j}}$ and $C_{S}$ is a dimensionless Smagorinsky coefficient is calculated from the instantaneous flow conditions using the dynamic determination procedure developed by Moin et al [22] for compressible flows, following the work of Germano et al [23]. The isotropic part of the SGS stress tensor in equation (9), $\tau_{k k}^{s g s}$ is modelled using the relation of Yoshizawa [24] as:

$$
\tau_{k k}^{s g s}=2 \bar{\rho} C_{I} \bar{\Delta}^{2}|\tilde{S}|^{2}
$$

where the model coefficient $C_{I}$ is very small and usually expected to be around 0.01 [15].

\subsection{Modelling of SGS scalar fluxes}

The Favre-filtered energy and reaction progress variable equations (5) \& (6) yields two unclosed terms, which must be closed using SGS modelling. The last terms on the LHS of equation (5) \& (6) are due to the scalar fluxes, similar to that of NS equation, which can be usually decomposed as $\bar{\rho} \overline{u_{j}^{\prime \prime} h^{\prime \prime}}=\bar{\rho}\left(\widetilde{u_{j} h}-\tilde{u}_{j} \tilde{h}\right)$ and $\bar{\rho} \overline{u_{j}^{\prime \prime} c^{\prime \prime}}=\bar{\rho}\left(\widetilde{u_{j} c}-\tilde{u}_{j} \tilde{c}\right)$ respectively. Modelling of these SGS scalar fluxes in turbulent reacting flames is highly challenging due to their non-linear relation with chemical and thermodynamic states. The major difficulty in modelling is due to the anisotropic behaviour of scalar fluxes. This is strongly affected by the turbulent velocity fields, through the large increase in specific volume and viscosity, which causes large temperature rise in reacting flows. Modelling could be further complicated due to the large pressure gradients and density variations associated with heat release, which may cause non-gradient transport (NGT) or counter gradient diffusion.

Boger and Veynante [25] identified that in LES of turbulent premixed flames, the simple gradient transport assumption is able to recover counter gradient transport at resolved scales without any extra effort. Hence, the present work uses the simple 
gradient transport model presented in equations (5) \& (6) for scalar fluxes. It should be noted here that the effect of counter gradient transport is still significant and the model presented in Knikker et al [4] may predict much accurately. However, this fact is not tested in the present work.

$$
\begin{gathered}
\bar{\rho} \overline{u_{j}^{\prime \prime} h^{\prime \prime}}=-\frac{\bar{\mu}_{\mathrm{SGS}}}{\operatorname{Pr}_{t}} \frac{\partial \tilde{h}}{\partial x_{j}} \\
\bar{\rho} \overline{u_{j}^{\prime \prime} c^{\prime \prime}}=-\frac{\bar{\mu}_{\mathrm{SGS}}}{\mathrm{Sc}_{t}} \frac{\partial \tilde{c}}{\partial x_{j}}
\end{gathered}
$$

where $\mathrm{Pr}_{\mathrm{t}}$ is the turbulent flow Prandtl number and $\mathrm{Sc}_{t}$ is the turbulent flow Schmidt number.

\subsection{Modelling of mean chemical reaction rate}

Similarly, the chemical reaction rate i.e. the last term on RHS of equation (6) can be modelled by either a simple Eddy-Break-Up (EBU) [26] assumption which gives a reaction rate proportional to the time scale of turbulent mixing or by using more advanced models based on the FSD [8]. The FSD approach is mainly based on the laminar flamelet concept of expressing the mean reaction rate per unit volume, $\overline{\dot{\omega}}_{c}$ as

$\overline{\dot{\omega}}_{c}=R \bar{\Sigma}$. Here $R$ is a mean reaction per unit surface area and $\bar{\Sigma}$ is the flame surface density (the flame surface area per unit volume), which is either modelled [7] or obtained by solving a full transport equation [27]. Solving transport equation for flame surface density [28] is an attractive option in LES, but results in several unclosed terms which need to be closed by appropriate models and lead to excessive computational cost.

On the other hand, mean chemical reaction rate per unit surface area $R$ can be written as $\rho_{u} u_{L}$, where $\rho_{u}$ is the density of unburned mixture and $u_{L}$ is the laminar flame velocity. Following the DNS analysis of thin premixed flames [14], flame surface density is calculated as: 


$$
\bar{\Sigma}=4 \beta \frac{\tilde{c}(1-\tilde{c})}{\bar{\Delta}}
$$

where $\tilde{c}$ is Favre filtered reaction progress variable, $\bar{\Delta}$ is the filter width and $\beta$ is the model coefficient developed from the DNS analysis [14]. The above expression is similar to the Bray-Moss-Libby (BML) expression for flame surface density in RANS [29]. The ratio $\bar{\Delta} / \beta$ represents the degree of sub-grid scale flame wrinkling.

The model coefficient $\beta$ is not universal and known to depend on many physical parameters such as filter width, chemistry and turbulence levels. Hence, in the present study, an additional run has been carried out with a sub-model for model coefficient in equation (15). This facilitates to verify the influence of the model coefficient on the LES solution. Following the work of Charlotte et al [2] we have identified that $\beta$ is of multiplicative in nature and applying Germano identity [23] to equation (15) will fails. By viewing the propagating flame as a wrinkled fractal surface, the model coefficient can be calculated using a wrinkling flame factor coupled with the fractal theory, as described in the following section.

\subsection{A sub-model for model coefficient,}

From the DNS analysis of the FSD [14], it is identified that the model coefficient $\beta$, represents the flame wrinkling factor in the FSD equation (16) shown below. In equation (16), $\alpha$ is a model constant and $\Xi$ is flame wrinkling factor. However in the present study, the flame wrinkling factor $\Xi$ is calculated locally from its definition in order to enhance the accuracy of the solution.

$$
\bar{\Sigma}=4 \beta \frac{\tilde{c}(1-\tilde{c})}{\bar{\Delta}}=4 \alpha \Xi \frac{\tilde{c}(1-\tilde{c})}{\bar{\Delta}}
$$

Defining the flame wrinkling factor $\Xi$ in the above equation, as a ratio of flame surface density to its projection in the normal direction of the flame propagation [2] and identifying the flame surface as a fractal surface between the inner and outer cut-off scales leads to: 


$$
\Xi=\left[\frac{\bar{\Delta}}{\delta_{c}}\right]^{D-2}
$$

This approach was successfully implemented in the thickened flame modeling [2]. In the above equation (17), the inner cut-off scale $\delta_{c}$ corresponds to the smallest scales of wrinkling flame and the outer cut-off scale $\bar{\Delta}$ represents the filter width corresponding to the grid resolution. In choosing inner cut-off scales, there are several hypotheses available based on physical arguments such as use of thee Kolmogorov length scale [30], the Gibson scale [31] and the laminar flame thickness [32].

Kolmogorov scale is the smallest physical length scale in any turbulent flow and is widely exploited by Gouldin [30], who identified that smoothing action in numerical simulations will generally wipe out the information regarding smallest scales and increases the inner cut-off scale. Also, at high values of $u^{\prime} / u_{L}$, the Kolmogorov scale can become smaller than the laminar flame thickness [33]. Where as, Peters [31] identified that the Gibson scale as the smallest scale, which remains in the reaction rate region long enough to alter the flame structure and is appropriate to consider as inner cut-off scale. However, Murayama and Takeno [32] argued that it is impossible for an object to have wrinkles smaller than its thickness, which eventually implies laminar flame thickness should be appropriate to consider as inner cut-off scale while using laminar flamelet concepts. Experimental studies of Gülder and Smallwood [34] supports this concept by relating inner cut-off scale as, $\delta_{c} \propto \delta_{L}$. This fact has been recently exploited by using inner cut-off scale equals to three times of laminar flame thickness [4]. Accordingly, a lower cut-off scale equal to three times of laminar flame thickness is used in the present work.

The fractal dimension $D$ in equation (17) is calculated by assuming the flame surface as a fractal surface in 3D space and is calculated by the following empirical relation [33]:

$$
D=\frac{2.19}{\left(\frac{u_{\Delta}^{\prime}}{u_{L}}+1\right)}+\frac{2.35}{\left(\frac{u_{L}}{u_{\Delta}^{\prime}}+1\right)}
$$


It should be noted that, the model constant $\alpha$ in equation (16) is considered as unity in the present simulation.

\section{The LES Calculations}

The calculations have been performed using a compressible version [15] of the LES code PUFFIN originally developed by Kirkpatrick [35] and used to predict turbulent flames by Masri et al [1]. PUFFIN solves strongly coupled Favre-filtered flow equations outlined above, which are written in a boundary fitted co-ordinates and discretized by using a finite volume method. The discretization is based on control volume formulation on a staggered non-uniform Cartesian case. A second order central difference approximation is used for the diffusion, advection and pressure gradient terms in the momentum equations and for gradient terms in the pressure correction equation. Conservation equations for scalars use second order central difference scheme for diffusion terms. The third order upwind scheme SHARP is used for advection terms of the scalar equations to avoid problems associated with oscillations in the solution. The QUICK scheme is used sometimes for the momentum equations in areas of the domain where the grid is expanded and accurate calculation of the flow is less important.

The equations are advanced in time using the fractional step method. Crank-Nicolson scheme is used for the time integration of momentum and scalar equations. A number of iterations are required at every time step due to strong coupling of equations with one other. Solid boundary conditions are applied at the bottom, vertical walls, for baffles and obstacle, with the power-law wall function of Werner and Wengle [36] used to calculate wall shear. Outflow boundary conditions are applied at the vented end of chamber. A non-reflecting boundary condition [35] analogous to commonly used convective boundary condition, in incompressible LES is used to prevent reflection of pressure waves at this boundary. The initial conditions are quiescent with zero velocity and reaction progress variable. Ignition is modelled by setting the reaction progress variable to 0.5 with in the radius of $4 \mathrm{~mm}$ at the bottom centre of the chamber. 
The discretized equations described above, are solved using a Bi-Conjugate Gradient solver with an MSI pre-conditioner for the momentum, scalar and pressure correction equations. The time step is limited to ensure the CFL number remains less than 0.5 with the extra condition that the upper limit for $\delta t$ is $0.3 \mathrm{~ms}$. The solution for each time step requires around 8 iterations to converge, with residuals for the momentum equations less than 2.5e-5 and scalar equations less than 2.0e-3. The mass conservation error is less than $5.0 \mathrm{e}-8$.

Simulations were carried using a three dimensional, non-uniform, Cartesian co-ordinate system for compressible flow with low Mach number. Since this type of flow involves large changes in density, high velocities and significant dilatation, all terms in the governing equations are retained. All calculations are performed on a Viglen Genie computer with a Xeon ${ }^{\circledR}$ processor, requiring approximately 3Gb RAM. Typical running times for various grids employed are detailed in Table 1.

\subsection{Computational domain}

The computational domain has the dimensions of 50x50x250 mm (combustion chamber) where the combustion takes place over the built-in baffles and solid obstruction, surrounded by solid wall boundary conditions. This domain is extended adequately to $325 \mathrm{~mm}$ in $x, y$ and $250 \mathrm{~mm}$ in $z$ directions with the far-field boundary conditions. LES simulations are carried out for five cases with four grid resolutions as detailed in Table 1.

\section{Results and Discussion}

One of the main objectives of this paper is assessing the LES solution and establishing confidence, such that the drawbacks can be identified for further modelling. The LES simulations of turbulent premixed propagating flames presented in this work are using four different grid resolutions as detailed in Table 1 with model coefficient, $\beta=1.2$ and using a sub-model for model coefficient, $\beta$. 
The time histories of the overpressure for four LES cases i.e. A, B, C and D near the closed ignition end of the chamber are considered and plotted together with experimental measurements [6] as shown in Figure 3. Cases A and B shows an initial increase in pressure at 5 and $6 \mathrm{~ms}$ after ignition, while this instance corresponds to $8 \mathrm{~ms}$ for cases C and D. Case B has predicted the correct peak overpressure incidence time as 10.2 ms compared to the experimental time and however, under-predicted the overpressure. It is also evident from Figure 3 that, case C (finer than grid B) has produced totally a different solution than case B. Since, LES solution B is not grid independent, it is not appropriate to consider for further evaluation.

Both the cases $\mathrm{C}$ and $\mathrm{D}$ has predicted the peak overpressure occurrence at $11.1 \mathrm{~ms}$ against $10.2 \mathrm{~ms}$ of experimental time. Similarly the magnitude of peak overpressure has predicted as 110 and 102 mbar against 138 mbar of experimental measurement. However, it can be noticed from Figure 3, that the increase in the overpressure after quasi-laminar phase of propagating flame in cases $\mathrm{C}$ and $\mathrm{D}$ corresponds to the time, where the flame is about to interact with the baffle plate (see Figure 1) as seen in experiments. Hence, LES solutions from cases C and D can be considered as grid independent based on overpressure trend and the time of occurrence of peak, even though there is a minor difference in its peak value. Also, the overpressure gradient is in reasonable agreement with experimental measurements and considered here for further evaluation. Nevertheless, it should be noted that the peak overpressure and its time of occurrence are under-predicted irrespective of grid refinement considered. The main reasons for these under-predictions are also explained in the following sections.

\subsection{Influence of the filter width, $\bar{\Delta}$}

The accuracy of the solution in LES is associated with several numerical and modelling parameters such as grid spacing $h$, filter width $\bar{\Delta}$, discretization schemes, solver, initial and the boundary conditions employed. For a given discretization scheme, solver, initial and the boundary conditions, the remaining critical numerical parameters that affect the LES solution are the grid spacing and the filter width. Filtering the flow field for large eddies by choosing an appropriate and optimal filter width does resolve the maximum 
amount of turbulent kinetic energy. In the present study, a box filter given in equation (2) is used.

In a conceptual study, Pope [16] hypothesizes that the LES solution may reach an intermediate asymptote when the filter width lies within the inertial sub-range. The relation between grid spacing and filter width was studied as a ratio of $h / \bar{\Delta}$ by Vreman et al [37] and Chow and Moin [38] for non-reacting cases. Their studies figured out the fact that the small values of $h / \bar{\Delta}$ correspond to excellent numerical accuracy and the higher values correspond to resolving a greater range of turbulence motions with less numerical accuracy. Vreman et al [37] and Chow and Moin [38] identified that, with a specified SGS model for turbulence, $h / \bar{\Delta}<=0.25$ with second order spatial accuracy or $h / \bar{\Delta}<=0.5$ with sixth order spatial accuracy has given numerically accurate solutions. Examination of this fact in case of reacting flows is computationally very expensive and requires an experimentally validated DNS solution and found no studies reported till date.

In the present work modest attempt has been made to examine the dependency of the numerical accuracy on the filter width. We considered two important ratios associated with the filter width. Firstly, grid spacing to the filter width and the second is, filter width to the laminar flame width. Grid spacing, $h$ in the present study is not homogenous and generally varies in the flame propagating direction (z-axis). Therefore all the relevant estimates used here are calculated using the grid spacing in the flame propagation direction and are presented in Table 2.

For cases $\mathrm{A}, \mathrm{B}, \mathrm{C}$ and $\mathrm{D}$, the $h / \bar{\Delta}$ ratio is plotted against filter width as shown in Figure 4(a). Correlating Figures 3 and 4(a) clarifies the fact, that the accuracy of solution in terms of generated overpressure has been improved as $h / \bar{\Delta}$ ratio tends to zero. Considering the $h / \bar{\Delta}$ ratio from the Table 2 for cases A, B and D, it is clear that they are almost all identical and however, the solution D is in better agreement. The $h / \bar{\Delta}$ ratio for case $C$ is $0.5-0.6$ and is different from the values of case $D$ as $0.37-0.5$. It is evident from Figure 3 that both cases $\mathrm{C}$ and D has predicted similar overpressure trend with minor differences. It should be noted here, that a small change in $h / \bar{\Delta}$ ratio is 
affecting the numerical accuracy of the solution. However, it is not yet clear, which factor i.e. $h$ or $\bar{\Delta}$ is dominating in deciding the accuracy of the solution. This is mainly due to the implicit nature of the filter width used in the present simulations.

In order to shed more light, let us consider the second ratio i.e. filter width to the laminar flame thickness $\left(\bar{\Delta} / L_{f}\right)$. For case $\mathrm{A}$ it ranges from 10.8 to 13.6 , and for case $\mathrm{B}$ it ranges from 8.78 to 10.6 compared to 5.10 to 5.16 for case $C$ and 4.40 to 5.10 for case D. Here $L_{f}$ is the calculated strained laminar flame thickness and this is different from the unstrained laminar flame thickness, $L_{f 0}$ which is a specified input parameter $\left(L_{f 0}=\right.$ $0.3 \mathrm{~mm}$ ). Correlating Figure 4(b) with Figure 3, it should be noted that the accuracy of the solution has improved as the $\bar{\Delta} / L_{f}$ ratio diminishes. It should be mentioned at this stage that, as $\bar{\Delta} / L_{f}$ changes from 5.10 to 5.16 in case $\mathrm{C}$ to 4.40 to 5.10 in case $\mathrm{D}$, computational cost of the solution has almost all doubled. Hence, it is essential to ensure that the filter width remains sufficiently larger than the strained laminar flame thickness. It can be seen from the estimates presented in Table 2 that $L_{f}$ is varying very slightly with the turbulence levels for the propane/air flames discussed in this work. Hence, the

filter width, $\bar{\Delta}$ is the main and only critical parameter on which accuracy depends.

From the above analysis, the filter width is identified to be a key factor in assessing the numerical accuracy. Since, the filter width is implicitly connected with the grid resolution in the current approach, it is very difficult to come to a conclusion regarding the appropriate ratio of $h / \bar{\Delta}$ in reacting cases. However, the filter width is a crucial factor in determining the resolved turbulent kinetic energy for a good LES, which is also examined in the next section.

\subsection{Resolved turbulent kinetic energy}

Based on the definition of LES, the quality of any LES simulation is dependent on the percentage of the resolved turbulent kinetic energy. For high Reynolds number flows, Celik et al [39] considered that resolving 70 to $85 \%$ of turbulent kinetic energy is sufficient and can be treated as a good LES. Encouragingly, Kempf et al. [40] tested this fact for LES of non-premixed flames over bluff bodies. In the present work, the quality 
of LES simulation is tested by calculating the resolved and modelled kinetic energy for cases $\mathrm{C}$ and $\mathrm{D}$ at various realizations.

The resolved turbulent kinetic energy in the present simulations is calculated by deducing the velocity fluctuations in $x, y$ and $z$ directions as $u^{\prime}, v^{\prime}$ and $w^{\prime}$ respectively. The difficulty aroused in the present case is due to the transient nature of the flame-flow interactions in calculating fluctuations from the instantaneous values. In case of LES, the data is available from only one simulation and the ensemble averaging, generally used on experimental data is not suitable to extract the velocity fluctuations. Hence, the only alternative choice to calculate/obtain RMS of velocity is by choosing a suitable bin size. From LES calculations, it is identified that there exist large number of data points ( 100 to 500) for every one millisecond of flame propagation due to the limitation of CFL number. Therefore, a bin size of $0.5 \mathrm{~ms}$ has been chosen to extract average and RMS velocity as:

$$
\begin{gathered}
\bar{x}=\frac{\sum x_{i}}{n} \\
\mathrm{RMS}=\left(\frac{\sum\left(x_{n}-\bar{x}\right)^{2}}{n}\right)
\end{gathered}
$$

Once velocity fluctuations are known, resolved turbulent kinetic energy can be calculated as:

$$
k_{\text {res }}=\frac{1}{2}\left(u^{\prime 2}+v^{\prime 2}+w^{\prime 2}\right)
$$

As the SGS kinetic energy is not directly accessible, it is estimated according to Deardorff [41] and the SGS eddy viscosity in equation (11) as:

$$
k_{s g s}=\frac{1}{\left(C_{s} \bar{\Delta}\right)^{2}} v_{s g s}^{2}
$$

At any realization the "percentage of resolved turbulent kinetic energy, $\eta$ ” is calculated as:

$$
\eta=\frac{k_{r e s}}{k_{t o t}}=1-\frac{k_{s g s}}{k_{t o t}}
$$

where $k_{\text {tot }}$ is the total kinetic energy, which is summation of resolved and SGS kinetic energy. It is worth noting at the stage that $C_{s}$ is the dimensionless Smagorinsky 
coefficient and dynamically calculated values from the instantaneous flow conditions are used to evaluate modelled kinetic energy.

The percentage of resolved turbulent kinetic energy from case $\mathrm{C}$ and $\mathrm{D}$ at five instants between 8.0 to $10.0 \mathrm{~ms}$ as shown in Figure 5 is considered. The overpressure trend and rate of pressure rise during this period is overlapping for both the cases as shown in Figure 3, so this time-phase might have influenced the development of different overpressure peaks at later stages of flame propagation. Evidently it is observed that, both the LES grids have captured more than 70\% energy during these instants and the pattern of the resolved energy is more or less similar with very few differences.

Figure 5(c) presents the resolved turbulent kinetic energy spectrum at $10 \mathrm{~ms}$ from case C. The energy spectrum provided in Figure 5(c) is obtained by using the velocity auto correlation technique, which provides the estimation of the length of eddies in the domain. The estimated length scales are converted as wave number using $k=2 \pi / L$, where $L$ is the size of eddy obtained using auto correlation technique. The resulting energy spectrum plotted on log scale as in Figure 5(c) shows resolved turbulent energy within the inertial sub-ranges very well. It should also be noted that the slope of the spectrum is inline with the classical Kolmogorov’s energy spectrum.

\subsection{Results for flame structure, location, speed and pressure}

LES calculations of case $C$ are considered in the present section for further analysis of flame structure, location and speed. In order to facilitate a meaningful discussion, the combustion chamber is divided into five regions of interest to analyse the flame structure and to identify the regimes of combustion. Flame characteristics such as flame front location, speed and structure are extracted from experimental video images in order to validate the numerical predictions. Figure 6 shows the comparison for the flame location and speed for case C. From LES calculations, the flame location is obtained by locating the farthest position of the leading edge of the flame front from the bottom end (defined here as the most down stream location of the flame from the ignition point, where $\tilde{c}=0.5$ ). The flame speed is calculated from the rate of change over successive images of the flame location at the leading edge of the flame farthest from the ignition 
point. Numerically, the turbulent burning velocity is estimated by dividing the flame propagating speed with gas expansion ratio by assuming isobaric volume expansion of the burning gas (please see Ref [42] for more details). It should be noted here that the experimental measurements are analyzed from high speed video recorded (2000fps) images, where the time scale between two consecutive frames is $0.5 \mathrm{~ms}$, which is considered here as the bin size for relevant LES estimations.

From Figure 6(a) it is evident, that the flame position at various stages of the flame propagation is accurately predicted. Similarly from Figure 6(b \& c) it is identified that the flame speed either with respect to time or with respect to flame position using case $\mathrm{C}$ is reproduced well and is in very good agreement with experimental measurements. The calculated and measured data confirm that the peak overpressure occurs during the reconnection of the flame, downstream of the square obstacle in the blow down region. The peak overpressure induced during this stage is due to the trapped unburnt mixture around the square obstacle. It should be noted, however, that the peak overpressure is slightly under-predicted and there is a slight difference in the time of it occurrence.

It is also observed from the snap-shots of the predicted flame structure shown in Figure 7 and experimental flame images shown in Figure 8, that the flame goes through different phases (or regimes), of combustion while propagating through the chamber. To identify these phases, the progress and characteristics of the propagating flame is examined from ignition at the closed end until the exit of the chamber at other end. Three possible realizations in every region are considered from LES predictions to demonstrate the flame structure, wrinkled nature, turbulence levels and other flame characteristics as tabulated in Table 3. Due to the limitation of frame speed in case of experimental video images, it is not possible to compare LES snap-shots exactly at the same time reference. However, at least one experimental video image is considered from each region as shown in Figure 8. It should be noted at this stage that the resolved flame structure in case of LES generally requires 4 to10 grid points within the reaction zone depending on the flame location. 
5.3.1 Region1 (R1) This region is extended to $20 \mathrm{~mm}$ from the ignition point at the chamber closed end. In this region, the flame is thin and quasi laminar and propagates at almost the laminar burning velocity $\sim 0.45 \mathrm{~m} / \mathrm{s}$ until it start to approach the first baffle plate. This is confirmed from both numerical and experimental data shown in Figure 7(R1) \& 8(a) respectively.

5.3.2 Region 2 (R2) This region extends from 20 to $80 \mathrm{~mm}$ as shown in Figure 2, downstream of the ignition point. Within this region the flame propagates through the three baffle plates and traps a small amount of unburnt fuel/air mixture as it evolves from the baffle plates. The flame is then stretched further as it moves from one baffleplate after the other. The entrapment of the flame around the baffles and its evolution through jetting can be noticed from numerical and experimental images shown in Figure 7(R2) \& 8(b) respectively. From Table 3, a progressive increase from 1.0 to $4 \mathrm{~m} / \mathrm{s}$ of calculated turbulent burning velocities can be noticed.

5.3.3 Region 3 (R3) This region extends from 80 to $112 \mathrm{~mm}$ downstream from the ignition closed end. This region has a square obstacle of $12 \mathrm{~mm}$ sides. As seen in numerical and experimental images in Figure 7(R3) \& 8(c), the turbulent flame encounters square obstruction and propagates at a speed of $7.5 \mathrm{~m} / \mathrm{s}$ from the third baffle plate. This has lead to have a highly stretched and distorted flame as it interacts with the solid square obstacle and achieves a maximum of $9 \mathrm{~m} / \mathrm{s}$ of turbulent burning velocity. A rapid raise of overpressure from 40 to 70 mbar with a steep pressure gradient and a sharp increase in flame propagation speed from 15 to $50 \mathrm{~m} / \mathrm{s}$ is observed during this interaction.

5.3.4 Region 4 (R4) which extends from 112 to $150 \mathrm{~mm}$ downstream of ignition point. This region may be viewed as the start of the blow-down region where the flame starts exiting from the chamber. Due to the presence of the square obstacle in region 3, a significant amount of unburnt fuel/air mixture is trapped around the obstacle as shown in Figure 7(R4) \& 8(d). The flame is stretched further with an aim to reconnect the flame in recirculation zone and hence increases in area in this region. As a result, the 
pressure and the flame propagation speed found to increase further to 103 mbar and 80 $\mathrm{m} / \mathrm{s}$ respectively as the turbulent burning velocity increases to $10 \mathrm{~m} / \mathrm{s}$.

5.3.5 Region 5 (R5) covers the remainder of the chamber. Here, the blow-down phase continues where the flame propagates further to outside of the chamber and flame gets reconnected completely as shown in Figure $7(\mathrm{R} 5)$ \& 8(e). The overpressure is found to increase and achieves its maximum of 110 mbar further in this region due to the burning of the remaining fuel/air mixture trapped inside the chamber. Experimentally it is observed that the maximum overpressure is reached to 138 mbar by consuming the trapped mixture around the solid obstacles. It is also found that flame propagates at its maximum speed of around $140 \mathrm{~m} / \mathrm{s}$ driving towards the chamber exit. The generated pressure found to oscillate while the remaining trapped mixture is burning.

Relevant estimates from LES predictions at various instants of flame propagation with in the above regions are calculated and presented in Table 3. It is very interesting to note, that the level of agreement in case of flame position, propagating speed and the flame structure as shown in Figure 6, 7 and 8 at different instants are very convincing and confirming the validity of the LES predictions. Further to this, various regimes of combustion are calculated based on non-dimensional groups and identified them on two standard combustion regime diagrams as discussed in the following section.

\subsection{The regimes of combustion in the current chamber}

Data from LES simulations for case C is used to identify the regimes of combustion in the current chamber. Summary of the data extracted from LES calculations is presented in Table 3. All controlling parameters in this analysis are evaluated at the leading edge of the flame front as defined earlier. It should be noted here that this regime diagrams are not valid within the vicinity of wall. Firstly this is due to the scaling of turbulent length scales due to wall functions used and secondly, heat loss to the wall may lead to local quenching. 
The classical regime diagram for turbulent premixed flames as developed by Peters [17] is plotted for $u$ ' $u_{L}$ with $L_{I} / L_{f}$. An LES regime diagram for turbulent premixed flames has been developed further by Pitsch and De Lagenste [18] in terms of Karlovitz number (Ka) and the ratio of $\bar{\Delta} / L_{f}$. The objective of the current analysis is to identify the regimes of the flame at different stages of its propagation while interacting with the solid obstacles. Significant importance is given while the flame is ramming and evolving from the obstacles. The Karlovitz number is calculated here based on the filter width as stated by Pitsch and De Lageneste [18] as follows:

$$
\mathrm{Ka}=\left[\frac{u_{\Delta}^{\prime 3} L_{f}}{u_{L}^{3} \bar{\Delta}}\right]^{1 / 2}
$$

In order to verify other flow parameters, Reynolds numbers based on the integral length scale $\left(L_{I}\right)$, filter width $(\bar{\Delta})$ and Damköhler number based on filter width (Da) are calculated as follows.

$$
\begin{gathered}
\operatorname{Re}_{L I}=\frac{u_{\Delta}^{\prime} L_{I}}{v} \\
\operatorname{Re}_{\bar{\Delta}}=\frac{u_{\Delta}^{\prime} \bar{\Delta}}{u_{L} L_{f}} \\
\operatorname{Da}_{\bar{\Delta}}=\frac{u_{L} \bar{\Delta}}{u_{\Delta}^{\prime} L_{f}}
\end{gathered}
$$

The rms of turbulent velocity, $u$ ' is obtained [1] at the leading edge of the flame front and is calculated from:

$$
u_{\Delta}^{\prime} \approx \bar{\Delta}|\tilde{S}|=\bar{\Delta}\left(\left|2 \tilde{S}_{i j} \tilde{S}_{i j}\right|\right)^{1 / 2}
$$

The integral length scale $L_{I}$ is estimated to be $10 \%$ of the chamber width [1], i.e. $L_{I}$ is taken to be $5 \mathrm{~mm}$. The length scales and dimensionless number estimated as stated above at various time steps of the flame propagation are summarized in Table 3. Data from the LES simulation are plotted on the two regimes of combustion diagrams to get the adequate confirmation of the combustion model used in the present calculations as shown in Figure 9 (a \& b). Both regime diagrams confirm that the leading edge of the 
flame is always within the thin reaction zone irrespective of its position and interactions with the solid obstacles.

A rapid increase in the Karlovitz and Reynolds numbers is observed at the end of region1 (R1) where the flame starts approach the first solid baffle plate. This is confirmed by observing the abrupt increment of the flame speed from LES predictions (sudden hike from experimental measurements also can be clearly seen) from figure 4(d). The same trend of Karlovitz and Reynolds numbers can be observed until the flame starts evolving from the first baffle plate. A sudden fall in Karlovitz and Reynolds numbers is noticed as the flame propagates through first solid turbulence generating grid. At this point the flame is experiencing re-laminarisation due to the local flow conditions. As the first turbulence generating baffle is very near to the ignition end, it is not influencing much by the increase in turbulence level in the combustion chamber. Similar fluctuations trend of Karlovitz and Reynolds numbers is noticed as the flame propagates past second and third baffle plates. However the range of fluctuations of Karlovitz and Reynolds numbers is lowered as the turbulence level increases.

\subsection{Result using a sub-model for model coefficient,}

The calculation of the model coefficient $\beta$ as explained in Section 3.1 is implemented in LES code and examined for an additional run named as case E having 90x90x336 grid points in the domain. Results from LES simulations using case C with constant model coefficient and case E with a sub-model for model coefficient, in the flame surface density equation are compared against the experimental measurements and discussed in this section. For the initial analysis, time histories of the overpressure are shown in Figure 10(a). Solid line in Figure 10(a) represents numerical predictions using constant model coefficient and dash-dot line represents predictions using dynamic model for model coefficient, compared with solid line with square symbols representing experimental measurements. It is evident from Figure 10(a), that case E has predicted similar pressure trend and the rate of pressure rise to that of case C. However, slightly higher peak overpressure i.e. 114 mbar at 11.0 ms has been predicted with case E having a model for model coefficient compared with 110 mbar at $11.1 \mathrm{~ms}$ in case C. 
The overpressure trend from case $\mathrm{E}$ is slightly better than case $\mathrm{C}$ and however, still under-predicted.

Results for flame characteristics such as position and speed are shown in Figures 10 (b, c \& d). Evidently, Figure 10(b) shows a very good overlapping of the LES predictions with experiments, which are in excellent agreement. Figure 11 shows time histories for the model coefficient $\beta$, for both test cases, i.e. $\mathrm{C}$ and $\mathrm{E}$. The value of the model coefficient has been extracted from numerical data at the leading edge of the propagating flame front, where the flame location is defined. The solid line in Figure 11 represents the model coefficient as calculated by the sub-model and the thin dashed line represents results with constant $\beta$ value i.e. 1.2 used in simulations. It is noteworthy at this stage that, the model coefficient calculated by the wrinkling flame factor, is almost close to the constant value $(\beta=1.2)$ before reaching the peak overpressure i.e. $<11 \mathrm{~ms}$. After the peak overpressure, it can be noticed that the model coefficient has reached a maximum value of about 1.6, which is due to the implemented model. It can also be noticed that, the model coefficient has suddenly increased at around $11 \mathrm{~ms}$ from 1.2 to 1.25. This has caused a slightly higher SGS chemical reaction rate, leading to a little higher peak pressure.

Figure 11 also shows the fractal dimension $D$ (calculated from equation 18) represented by a dash-dot line showing on the right hand scale. These values are also extracted at the leading edge of the flame and representing the wrinkled nature of flame at various stages. The values of the fractal dimensions are confirming the dynamic nature of the flame wrinkling due to local turbulence, which has been captured at various instants. A sudden increase in the fractal dimension to 2.35 at around $8 \mathrm{~ms}$ is observed. This is where the flame protrudes through the third baffle plate and encounters the square solid obstacle. However, there is no much impact noticed due this increase on the model coefficient, as lower cutoff scale is acting as a damping function.

Overall, the results of test case E are very encouraging and confirm the advantage of using flame wrinkling model for the model coefficient, $\beta$. However it should be noted 
that at this stage the improvement in the predictions is not very significant and should be considered as an encouraging lead to implement the dynamic procedure for the calculation of the SGS flame surface density.

\section{Conclusions}

Large eddy simulations of premixed flame propagation inside a vented combustion chamber are reported in this paper. The simulations were performed for a stoichiometric propane/air mixture. LES simulations were carried out for various grid resolutions. LES predictions were examined and analyzed to understand the influence of filter width and the percentage of turbulent kinetic energy resolved. LES simulations were identified to predict overall turbulent premixed flame characteristics reasonable well with experimental measurements.

It can be concluded from the above study that the filter width is an important influencing factor in deciding numerical accuracy. However, it is identified that in case of implicit filtering, the chosen filter width is dependent on grid resolution and an agreement between these two is necessary in achieving sufficient numerical accuracy.

The turbulent kinetic energy resolved in the domain, has been identified to be mainly dependent of grid resolution, filter width and the Reynolds number. It was identified that the turbulent kinetic energy resolved in case of C and D is almost all same even though grid D is finer. This analysis shows that there is a limitation of grid employed on resolved turbulent kinetic energy. Also, the energy spectrum analyzed at $10 \mathrm{~ms}$ giving a good indication of energy distribution in inertial sub-range from large eddies to small eddies.

A detailed analysis has been carried out to determine the regime(s) of combustion in the chamber under study. The analysis is carried out at the leading edge of the flame fronts at various times after ignition. It is found that all regimes of combustions in the current 
test case lie within the thin reaction zone. This finding supports the use of the laminar flamelet modelling concept that has been in use for the modelling of turbulent premixed flames in practical applications.

Initially the under-prediction of overpressure was considered to be improved by a submodel for model constant $\beta$ in FSD equation. Accordingly, a model was developed and implemented in in-house LES code. A similar trend of all the flame characteristics such a position, speed and structure were identified with a little improvement in the predicted overpressure. The improvement in overpressure prediction has been identified as minimal and concluded as an encouraging lead to develop a dynamic flame surface density model by present authors.

\section{References}

[1] Masri, A.R., Ibrahim, S.S., and Cadwallader, B.J., 2006, Experimental Thermal and Fluid Science, 30, 687-702.

[2] Charlette, F., Meneveau, C., and Veynante, D., 2002, A power-law flame wrinkling model for LES of premixed turbulent combustion Part II: Dynamic formulation, Combustion and Flame, 131:181-197.

[3] Fureby, C., 2005, Proc. Combust. Inst., 30, 593-601.

[4] Knikker, R., Veynante, D., and Meneveau, C., 2002, Proc. Combust. Inst., 29, 21052111

[5] Pitsch, H., 2005, Combust. Flame, 143, 587-598.

[6] Kent, J.E., Masri, A.R., Starner, S.H., and Ibrahim, S.S., 2005, A new chamber to study premixed flame propagation past repeated obstacles. 5th Asia-Pacific Conference on Combustion, The University of Adelaide, Australia, 17-20.

[7] Bray, K.N.C., 1990, Studies of turbulent burning velocity, Proc. R. Soc. London, Ser., A431, 315.

[8] Pope, S.B., 1988, The evolution of surfaces in turbulence. Int. J. Engng. Sci., 26(5), 445-469. 
[9] Williams, F.A., 1985, In: Buckmaster, J., (Ed.) In The Mathematics of Combustion (SIAM: Philadelphia) 97-131.

[10] Kerstein, A., Ashurst, W., and Williams, F., 1998, Phys. Rev., A37 (7), 2728-2731.

[11] Menon, S., and Kerstein, A., 1992, Proc. Combust. Institute, 24, 443-450.

[12] Veynante, D., and Poinsot, T., 1997, Large eddy simulations of the combustion instabilities in turbulent premixed burners. Annual research briefs, Center for turbulence research. 253-275.

[13] Möller, S.I., Lundgren, E., and Fureby, C., 1996, Large eddy simulations of unsteady combustion. Proc. Combust. Institute, 26, 241.

[14] Boger, M., Veynante, D., Boughanem, H., and Trouve, A., 1998, Direct numerical simulation analysis of flame surface density concept for large eddy simulation of turbulent premixed combustion. Proc. Combustion Institute, 27, 917-925.

[15] Kirkpatrick, M.P., Armfield, S.W., Masri, A.R., and Ibrahim, S.S., 2003, Large Eddy Simulation of a Propagating Turbulent Premixed Flame, Flow turbulence and combustion, 70, 1-19.

[16] Pope, S.B., 2004, New Journal of Physics, 6, 35.

[17] Peters, N., 2000, Turbulent Combustion, Cambridge University Press.

[18] Pitsch, H., and De Lageneste, D., 2002, Proc. Combust. Inst., 29, 2001-2008.

[19] Damköhler, G.Z., 1940, Elektrochem., 46, 610.

[20] Pitsch, H., 2006, Large-Eddy Simulations of Turbulent Combustion. Annu. Rev. Fluid Mech., 38, 453-82.

[21] Smagorinsky, J., 1963, General circulation experiments with the primitive equations, I, The basic experiment. Monthly Whether Review, 91, 99-164.

[22] Moin, P., Squires, K., Cabot, W., and Lee, S., 1991, A dynamic subgrid-scale model for compressible turbulence and scalar transport. Physics of Fluids, A3, 27462757.

[23] Germano, M., Piomeli, U., Moin, P., and Cabot, W.H., 1991, A dynamic subgridscale eddy viscosity model. Phys. Fluids, A3(7), 1760-1765. 
[24] Yoshizawa, A., 1986, Statistical theory for compressible turbulent shear flows, with the application to subgrid modeling, Phys. Fluids, 29 (7), 2152-2164.

[25] Boger, M., and Veynante, D., 2000, Large eddy simulations of a turbulent premixed V-shaped flame, Advances in Turbulence VIII, 449.

[26] Spalding, D.B., 1971, Mixing and chemical reaction in steady confined turbulent flames. 13th Symposium (International) on Combustion, The Combustion Institute Pittsburgh, 649-657.

[27] Prasad, R.O.S., and Gore, J.P., 1999, Combust. Flame, 116, 1.

[28] Hawkes, E.R., and Cant, R.S., 2001, Implications of a flame surface density approach to large eddy simulation of premixed turbulent combustion. Combu. and Flame, 126, 1617-1629.

[29] Bray, K.N.C., Champion, M., and Libby, P.A., 1989, Flames in stagnating turbulence in Turbulent Reacting Flows (Eds.) Borghi, R., and Murphy, S.N., (Springer Publications: New York), 541- 563.

[30] Gouldin, F.C., 1987, An application of fractals to modeling premixed turbulent flames, Combustion and Flame, 68 (3), 249-266.

[31] Peters, N., 1988, Laminar flamelet concepts in turbulent combustion, Symposium (International) on Combustion, 21(1), 1231-1250.

[32] Murayama, M., and Takeno, T., 1989, Fractal-like character of flamelets in turbulent premixed combustion, Symposium (International) on Combustion, 22 (1), 551559.

[33] North, G.L., and Santavicca, D.A., 1990, Combust. Sci. Technol., 72, 215.

[34] Gülder, Ö.L., and Smallwood, G.J., 1995, Inner cutoff scale of flame surface wrinkling in turbulent premixed flames, Combustion and Flame, 103(1-2), 107-114.

[35] Kirkpatrick, M.P., 2002, A Large eddy simulation code for industrial and environmental flows. PhD Thesis, School of Aerospace Mechanical and Mechatronics eng. University of Sydney, Australia. 
[36] Werner, H., and Wengle, H., 1991, Large-eddy simulation of turbulent flow over and around a cube in a plate channel, 8th Symposium on Turbulent Shear Flows Munich, Germany.

[37] Vreman, B., Geurts, B. and Kuerten, H., 1996, Int. J. Numer. Meth. Fluids, 22, 297.

[38] Chow, F.K., and Moin, P., 2003, J. Comput. Phys., 184, 366.

[39] Celik, I., Cehreli, Z. N., and Yavuz, I., 2005, ASME, J. Fluids Eng., 127, 949-958.

[40] Kempf, A., Lindstedt, R.P., and Janicka, J., 2006, Large-eddy simulation of a bluff -body stabilized non-premixed flame, Combustion and Flame, 144, 170-189.

[41] Deardorff, J., 1980, Boundary-Layer Meteorol., 18, 495-527.

[42] Catlin, C.A., Fairweather, M., and Ibrahim, S.S., 1995, Predictions of Turbulent, Premixed Flame Propagation in Explosion Tubes, Combustion and Flame, 102, 115128. 
Table 1. Grid resolutions employed in the present study. $N_{x}, N_{y}$ and $N_{z}$ are the number of nodes in the $x, y$ and $z$ direction respectively.

\begin{tabular}{ccccccc}
\hline Case & $N_{x}$ & $N_{y}$ & $N_{z}$ & Model & Model Coefficient & Cost days \\
\hline A & 40 & 40 & 156 & FSD & 1.2 & 3 \\
B & 54 & 54 & 190 & FSD & 1.2 & 6 \\
C & 90 & 90 & 336 & FSD & 1.2 & 32 \\
D & 90 & 90 & 448 & FSD & 1.2 & 68 \\
E & 90 & 90 & 336 & FSD & Dynamic & 34 \\
\hline
\end{tabular}

Table 2. Details of the numerical parameters employed and results deduced from LES simulations for various grid resolutions for the configuration shown in Figure1.

\begin{tabular}{lllll}
\hline Parameters & A & B & C & D \\
\hline$\delta x(\mathrm{~mm})$ & 2.0 & 1.47 & 0.75 & 0.75 \\
$\delta y(\mathrm{~mm})$ & 2.0 & 1.47 & 0.75 & 0.75 \\
$\delta z(\mathrm{~mm})$ & $1.0-2.0$ & $1.0-1.75$ & $0.75-1.0$ & $0.48-0.75$ \\
$\bar{\Delta}(\mathrm{mm})$ & $3.17-4.0$ & $2.60-3.12$ & $1.5-1.65$ & $1.29-1.5$ \\
$h / \bar{\Delta}$ & $0.32-0.5$ & $0.39-0.56$ & $0.5-0.6$ & $0.37-0.5$ \\
$L_{f}(\mathrm{~mm})$ & 0.294 & 0.294 & 0.294 & 0.294 \\
$\bar{\Delta} / L_{f}$ & $10.8-13.6$ & $8.78-10.6$ & $5.10-5.61$ & $4.40-5.10$ \\
Peak pressure (mbar) & 85.6 & 96.7 & 110.0 & 102.0 \\
Time (ms) & 8.5 & 10.2 & 11.1 & 11.1 \\
Flame speed $(\mathrm{m} / \mathrm{s})$ & 62.3 & 88.3 & 81.8 & 81.0 \\
Flame position $(\mathrm{cm})$ & 12.0 & 17.8 & 17.9 & 18.2 \\
\hline
\end{tabular}


Table 3. Velocity and length scale estimates from LES predictions for case C. The flame structures at these times is produced from reaction rate contours as shown in Figure 7 and these estimates are fitted into known combustion regime diagrams as shown in Figure 9 (a) \& (b).

\begin{tabular}{lllllllllllll}
\hline & $\begin{array}{c}\text { Time } \\
(\mathrm{ms})\end{array}$ & $\begin{array}{c}u^{\prime} \\
(\mathrm{m} / \mathrm{s})\end{array}$ & $\begin{array}{c}u_{L} \\
(\mathrm{~m} / \mathrm{s})\end{array}$ & $\begin{array}{c}L_{f} \\
(\mathrm{~mm})\end{array}$ & $\begin{array}{c}u_{t} \\
(\mathrm{~m} / \mathrm{s})\end{array}$ & $u^{\prime} / u_{L}$ & $u_{t} / u_{L}$ & $\begin{array}{c}Z p \\
(\mathrm{~cm})\end{array}$ & $D a$ & $R e_{L i}$ & $K a$ & Regime \\
\hline \multirow{3}{*}{ R1 } & 3.5 & 2.10 & 0.450 & 0.294 & 0.45 & 4.66 & 0.99 & 1.181 & 1.10 & 1050 & 4.5 & TRZ \\
& 4.0 & 2.62 & 0.450 & 0.294 & 0.45 & 5.82 & 0.99 & 1.333 & 0.88 & 1310 & 6.2 & TRZ \\
& 4.6 & 4.43 & 0.450 & 0.294 & 0.51 & 9.84 & 1.125 & 1.562 & 0.53 & 2215 & 13.6 & TRZ \\
& 5.0 & 6.06 & 0.450 & 0.294 & 1.14 & 13.47 & 2.54 & 1.717 & 0.38 & 3030 & 21.7 & TRZ \\
R2 & 6.0 & 2.0 & 0.450 & 0.294 & 1.34 & 4.42 & 2.99 & 2.488 & 1.16 & 995 & 4.10 & TRZ \\
& 9.5 & 5.23 & 0.447 & 0.296 & 3.96 & 11.70 & 8.87 & 7.213 & 0.44 & 2615 & 17.7 & TRZ \\
& 9.8 & 4.55 & 0.447 & 0.296 & 6.27 & 10.18 & 14.02 & 8.283 & 0.50 & 2275 & 14.3 & TRZ \\
R3 & 10.0 & 4.0 & 0.446 & 0.296 & 7.80 & 8.90 & 17.50 & 9.040 & 0.57 & 1985 & 11.8 & TRZ \\
& 10.2 & 5.93 & 0.446 & 0.297 & 8.40 & 13.29 & 18.82 & 10.17 & 0.38 & 2965 & 21.5 & TRZ \\
& 10.3 & 5.63 & 0.445 & 0.297 & 8.69 & 12.65 & 19.54 & 10.70 & 0.40 & 2815 & 20.0 & TRZ \\
R4 & 10.6 & 4.78 & 0.444 & 0.298 & 9.58 & 10.77 & 21.57 & 13.03 & 0.47 & 2390 & 15.7 & TRZ \\
& 10.8 & 5.05 & 0.443 & 0.298 & 10.05 & 11.40 & 22.66 & 14.39 & 0.44 & 2525 & 17.1 & TRZ \\
& $11.1 *$ & 5.04 & 0.443 & 0.298 & 10.77 & 11.38 & 24.31 & 16.75 & 0.49 & 2520 & 16.3 & TRZ \\
R5 & 11.2 & 3.70 & 0.444 & 0.298 & 11.61 & 8.333 & 26.16 & 17.75 & 0.67 & 1850 & 10.2 & TRZ \\
& 11.3 & 4.90 & 0.444 & 0.298 & 12.46 & 11.04 & 28.05 & 18.75 & 0.51 & 2450 & 15.5 & TRZ \\
\hline
\end{tabular}

TRZ = Thin reaction zone

$\mathrm{Zp}=$ Flame position along z-axis

$*=$ Peak overpressure 


\section{Figure Captions}

[1] Schematic diagram of the premixed combustion chamber. All dimensions are in $\mathrm{mm}$.

[2] Region of interest along the combustion chamber. All dimensions are in $\mathrm{mm}$

[3] LES predictions of overpressure-time histories using various grid resolutions mentioned in Table 1 are compared with experimental measurements

[4] Estimates from LES simulation presented in Table 2 are plotted (a) Ratio of grid spacing to filter width is plotted versus filter width (b) Ratio of filter width to strained laminar flame thickness is plotter versus filter.

[5] Instantaneous percentage of resolved turbulent kinetic energy of turbulent premixed propagating flame at 8.0, 8.5, 9.0, 9.5 and $10.0 \mathrm{~ms}$ respectively. It can be noticed that more than $70 \%$ of total kinetic energy is resolved by the LES grid. (a) case C (b) case D. (c) The Resolved turbulent kinetic energy spectrum at $10 \mathrm{~ms}$ using grid C.

[6] LES predicted flame characteristics of the case $C$ is compared with the experimentally extracted data (a) Flame position (b) Flame speed and (c) Flame speed against flame position.

[7] Reaction rate images from case C, showing the flame propagation at different times after ignition with in the five regions. The time mentioned at the bottom of each chamber is in ms.

[8] Sequence of experimental images to show flame structure at different times after ignition (a) 4.5, (b) 6, (c) 10, (d) 10.5 and (e) $11.0 \mathrm{~ms}$.

[9] Estimates from case C of the LES simulations presented in Table 3 are fitted in to the regimes of combustions (a) Turbulent premixed combustion reported by Peters[9] (b) LES turbulent premixed combustion reported by Pitsch \& De Lageneste[11].

[10] Comparisons of LES simulations C and E with experimental measurements (a) Histories of overpressure (b) Flame position vs. time. 
[11] Time series of the model coefficient and fractal dimension using a sub-model in case $\mathrm{E}$ at the leading edge of the propagating flame. The dashed line is representing the constant model coefficient in case C. 


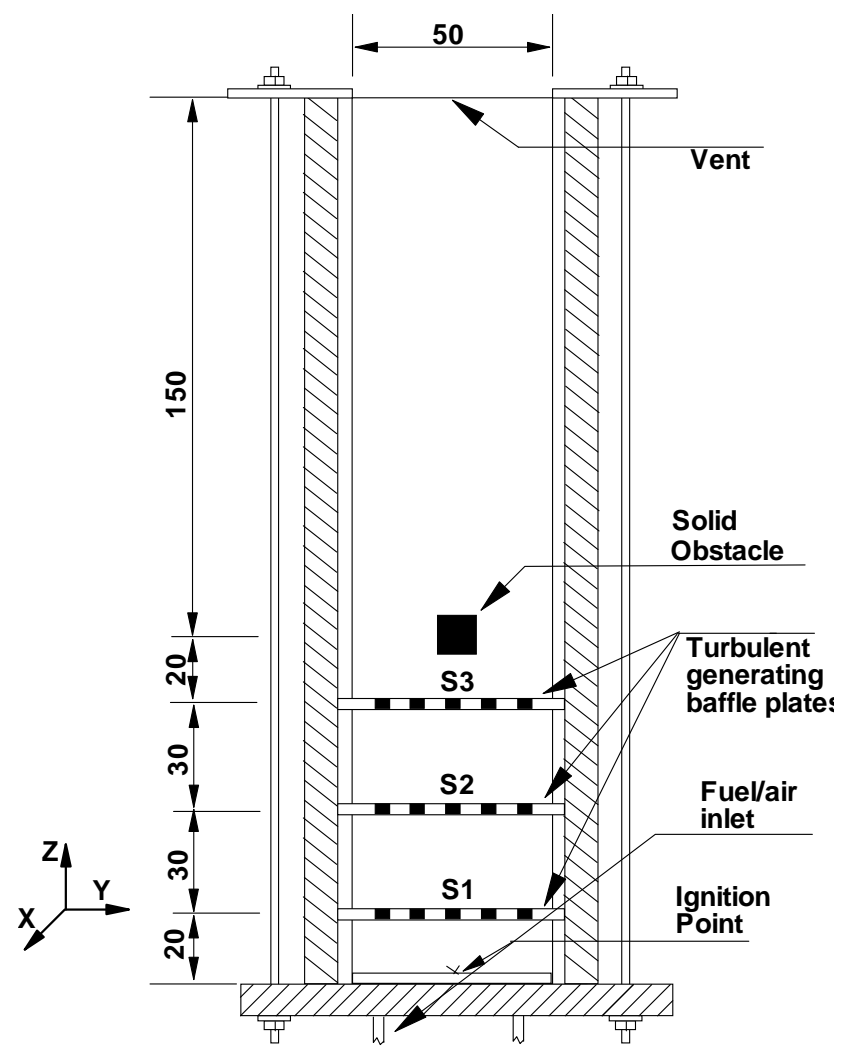

Figure 1. Schematic diagram of the premixed combustion chamber. All dimensions are in $\mathrm{mm}$.

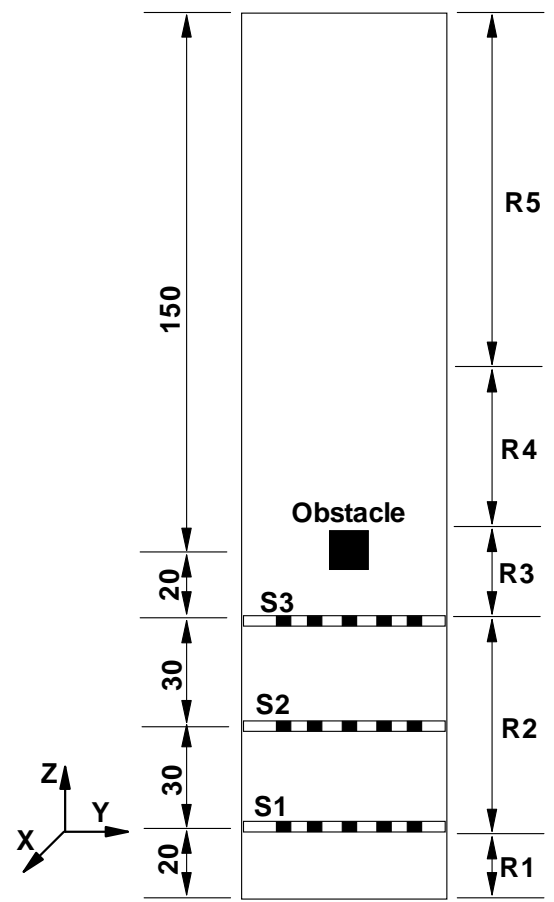

Figure 2. Region of interest along the combustion chamber. All dimensions are in mm. 


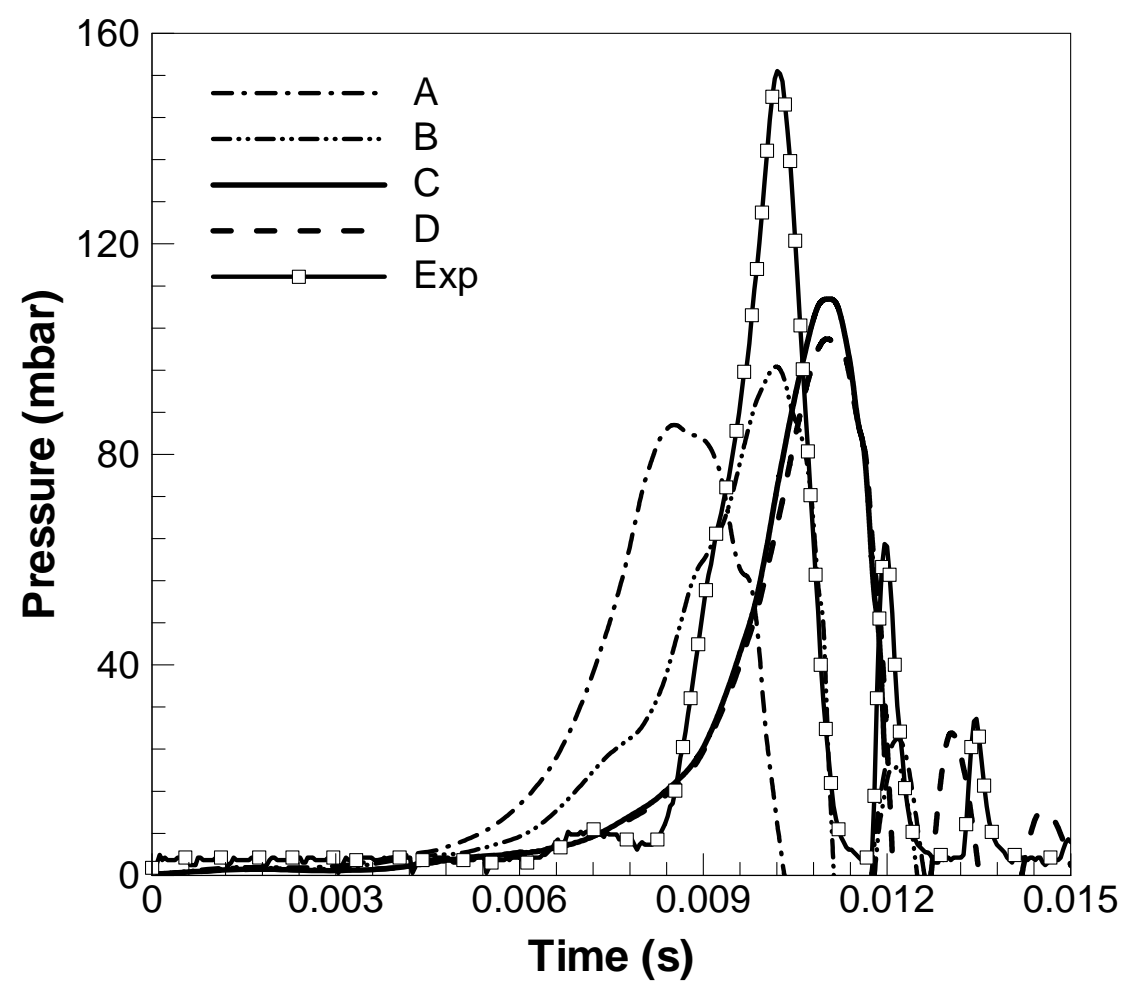

Figure 3. LES predictions of overpressure-time histories using various grid resolutions mentioned in Table 1 are compared with experimental measurements. 


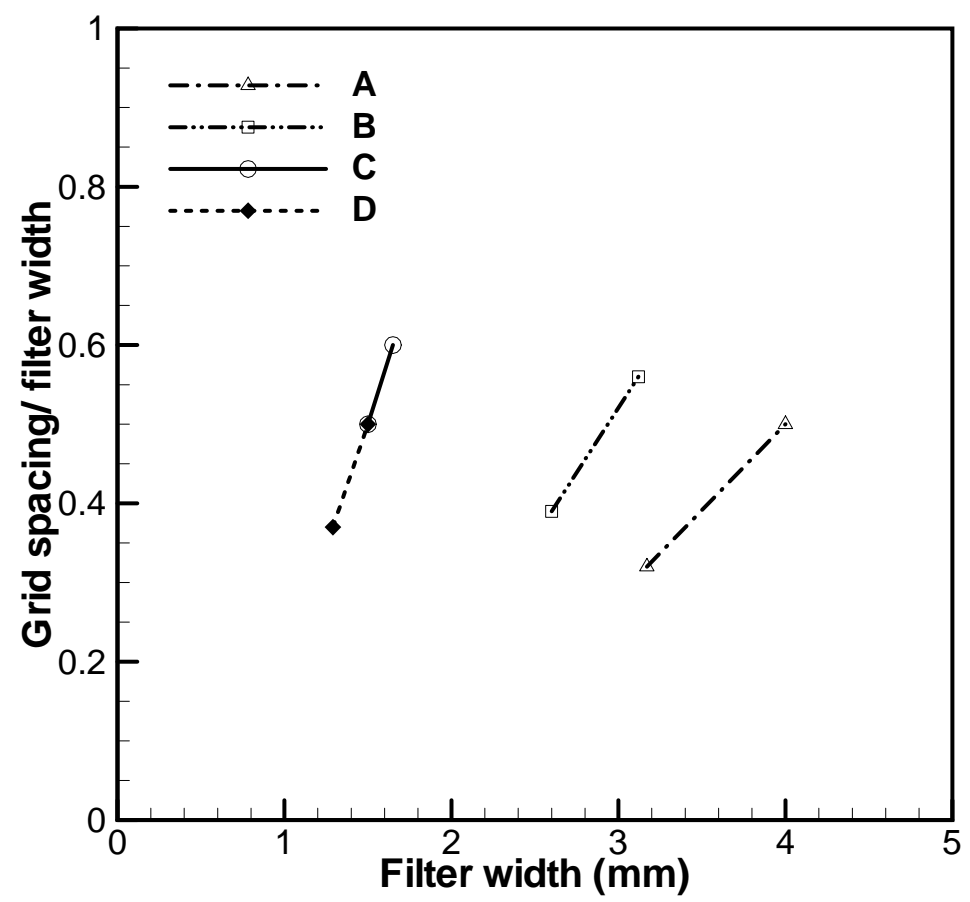

Figure 4a

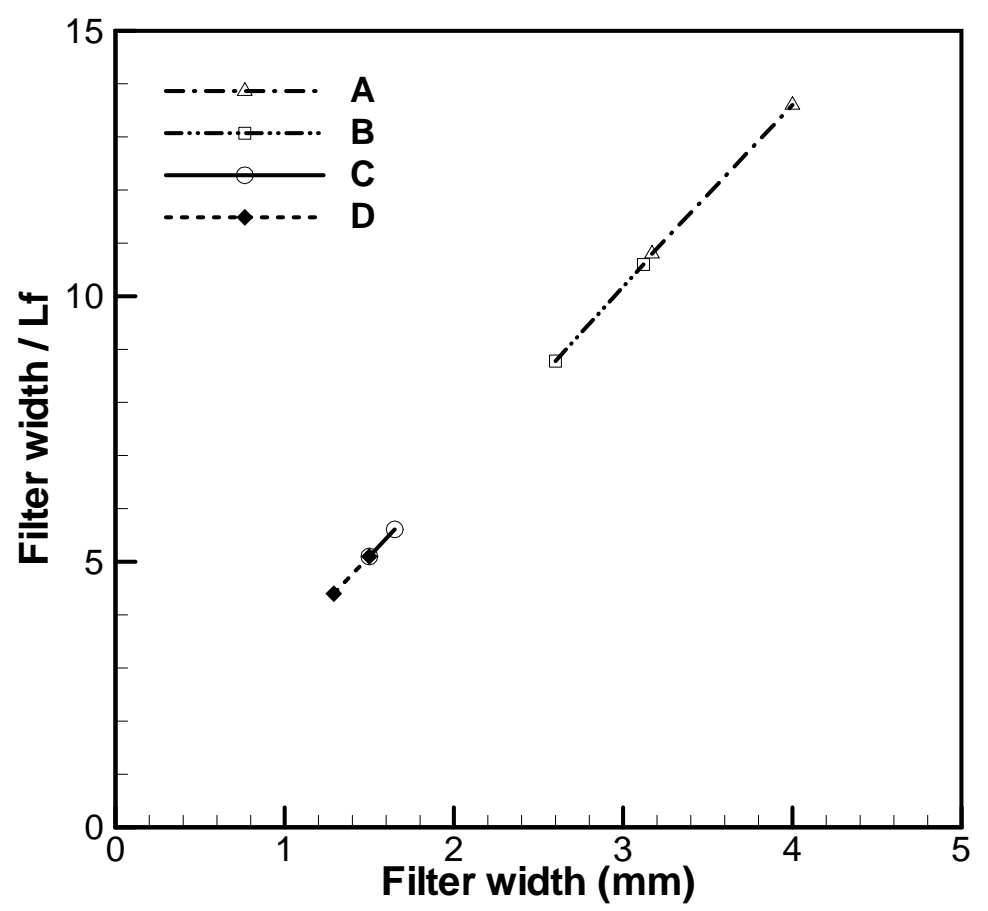

Figure $4 \mathrm{~b}$

Figure 4. Estimates from LES simulation presented in Table2 are plotted (a) Ratio of grid spacing to filter width is plotted versus filter width (b) Ratio of filter width to strained laminar flame thickness is plotter versus filter width. 


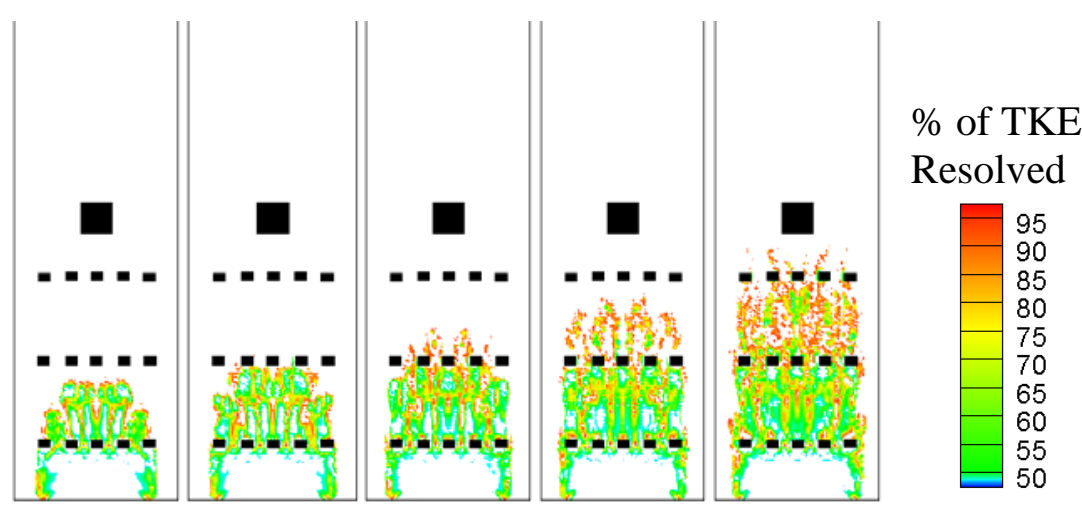

(a)

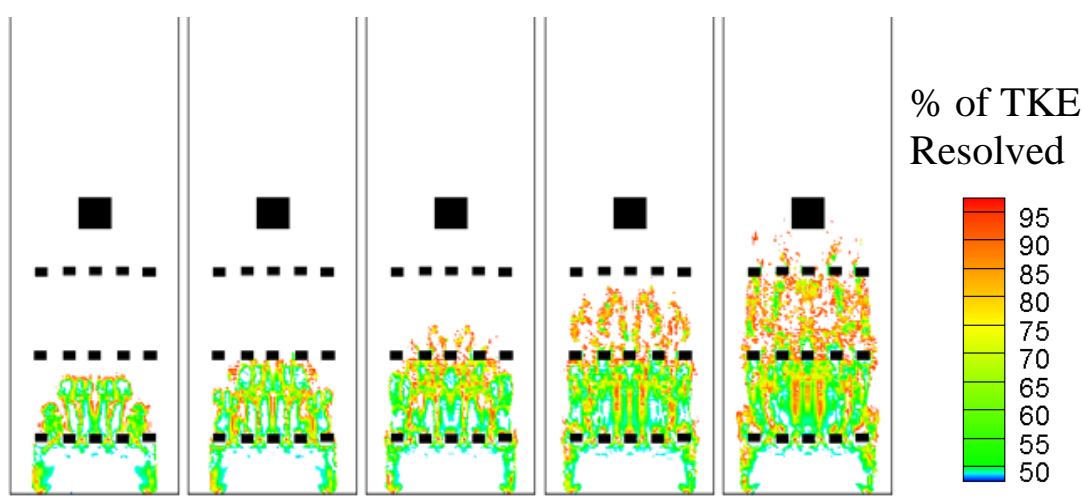

(b)

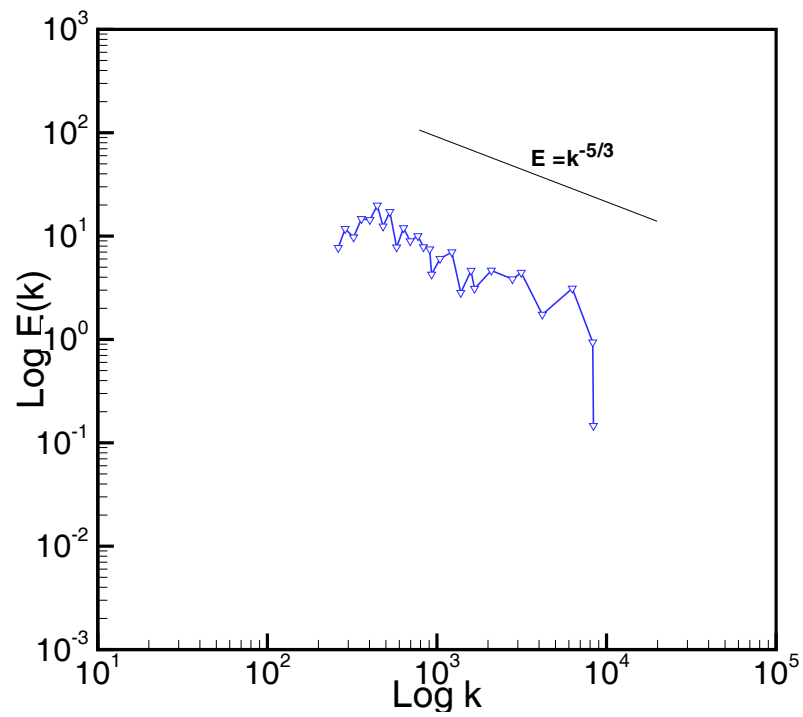

(c)

Figure 5. Instantaneous percentage of resolved turbulent kinetic energy of turbulent premixed propagating flame at 8.0, 8.5, 9.0, 9.5 and 10.0 ms respectively. It can be noticed that more than $70 \%$ of total kinetic energy is resolved by the LES grid. (a) case C (b) case D. (c) The Resolved turbulent kinetic energy spectrum at $10 \mathrm{~ms}$ using grid C. 


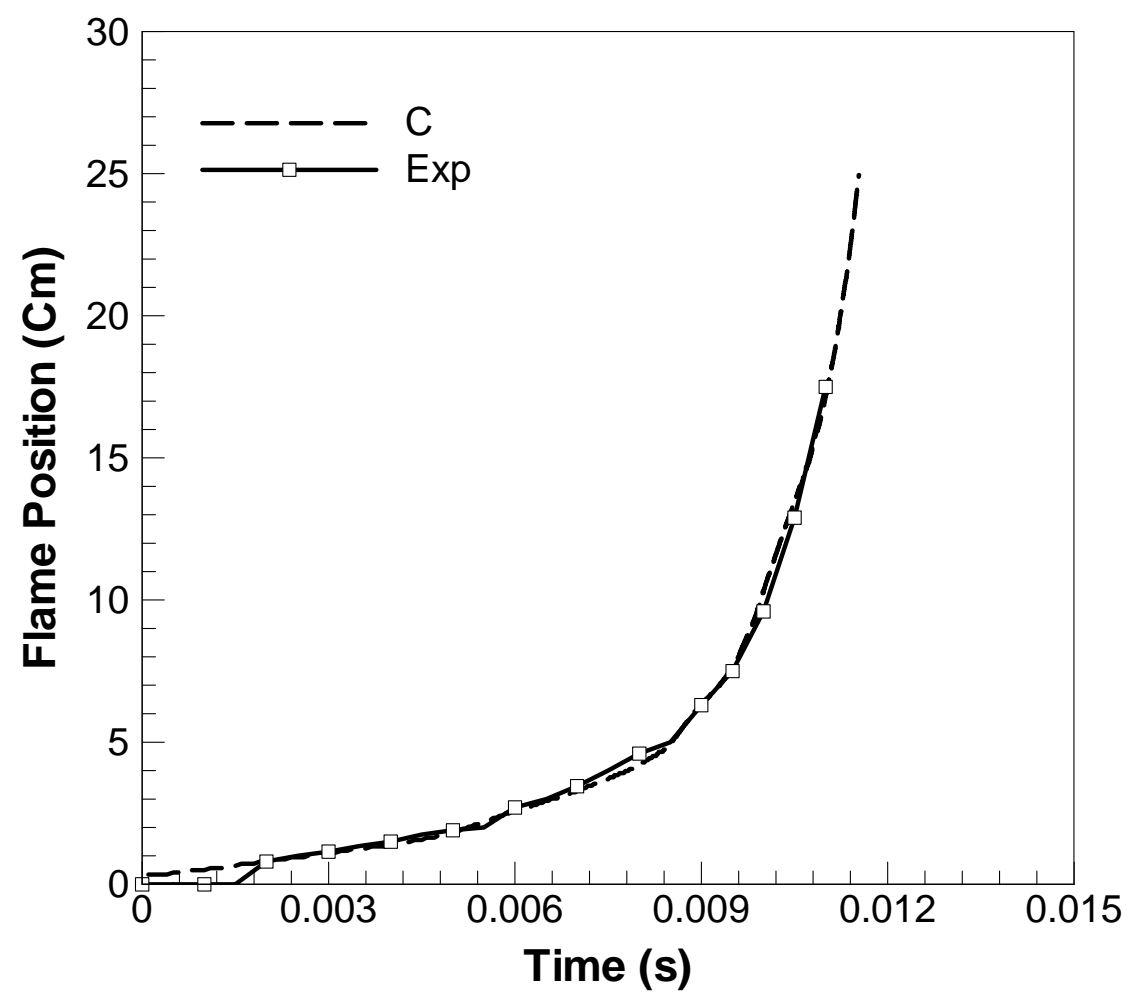

Figure 6a

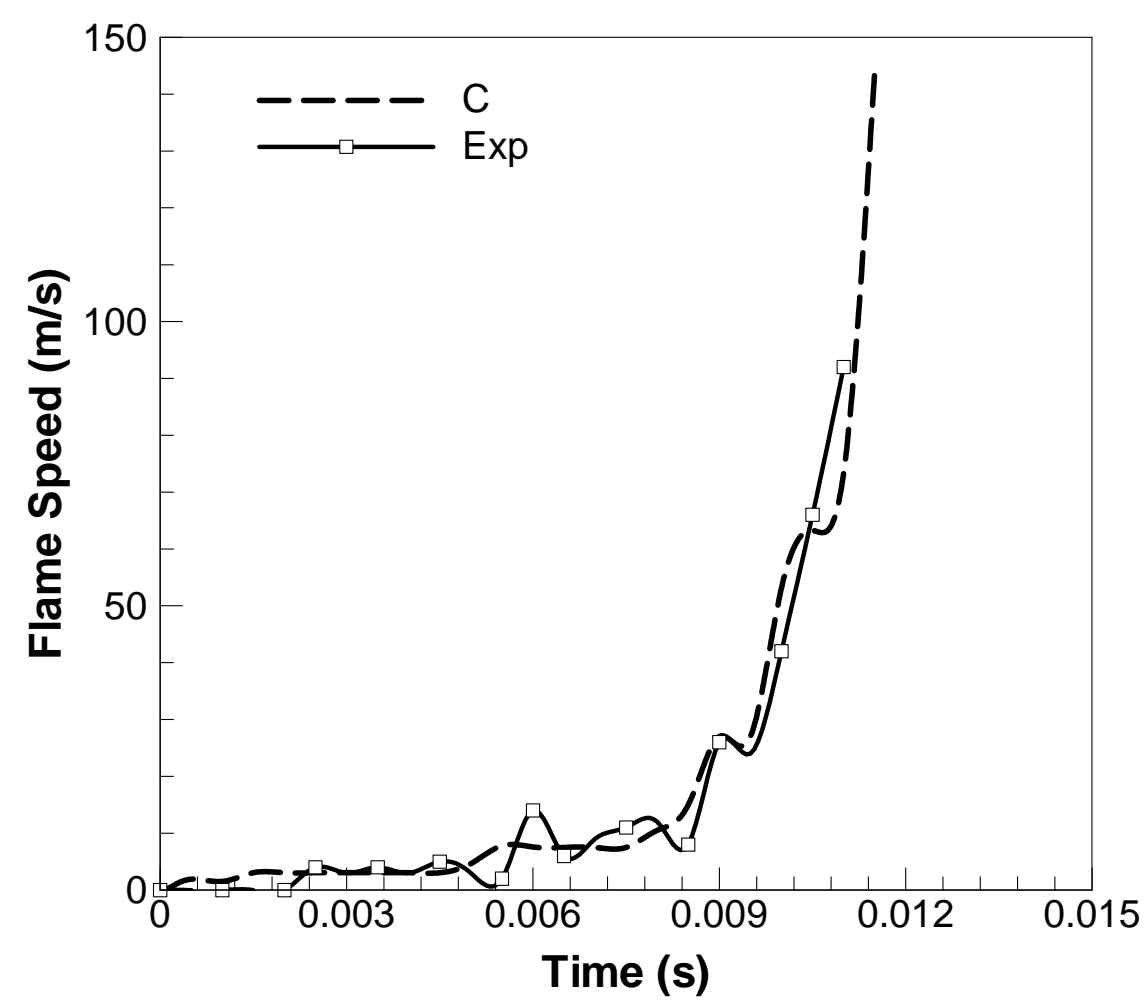

Figure $6 b$ 


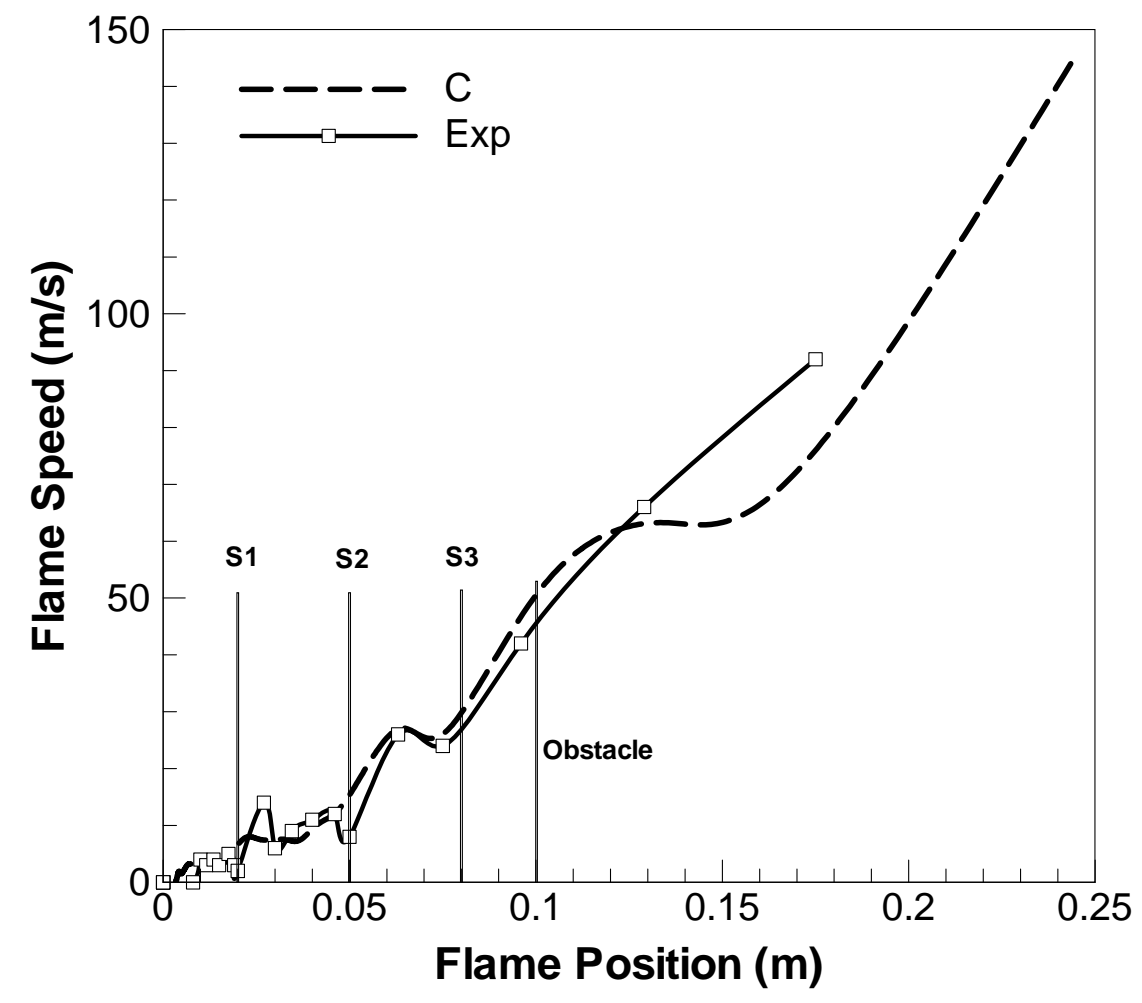

Figure 6c

Figure 6. LES predicted flame characteristics of the case $C$ is compared with the experimentally extracted data (a) Flame position (b) Flame speed and (c) Flame speed against flame position. 

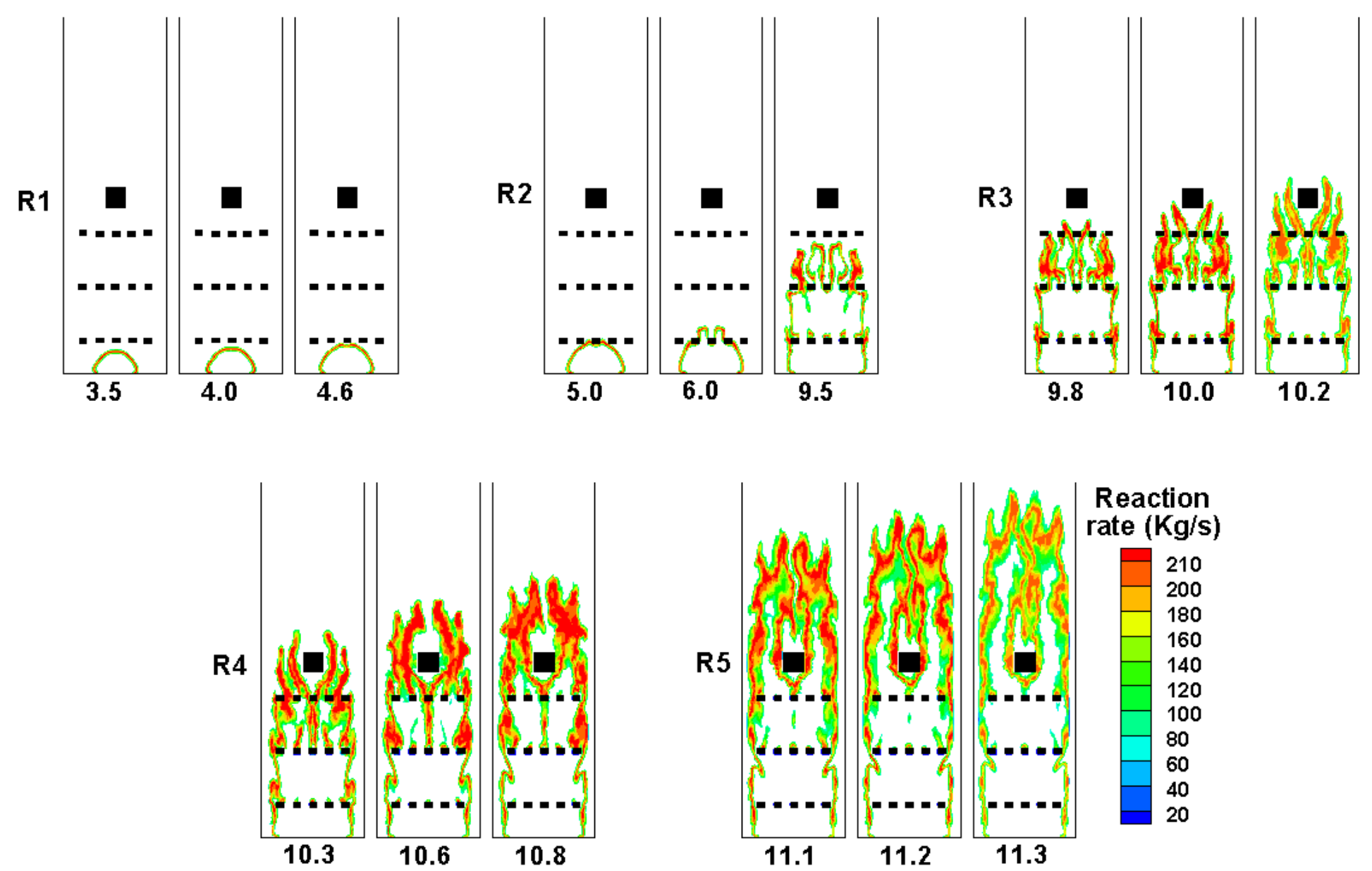

Figure 7. Reaction rate images from case C, showing the flame propagation at different times after ignition with in the five regions. The time mentioned at the bottom of each chamber is in ms. 


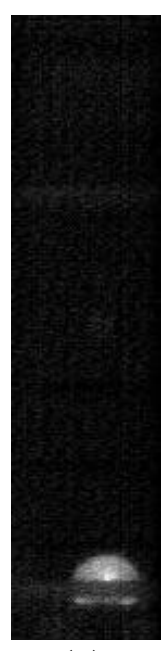

(a)

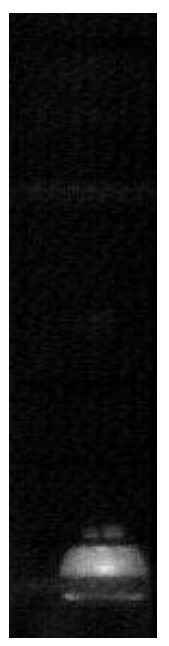

(b)

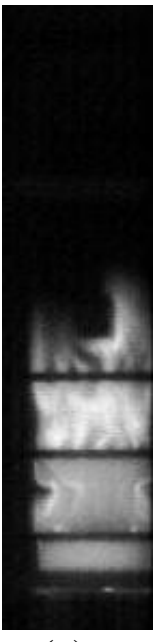

(c)

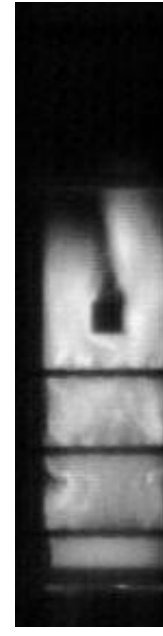

(d)

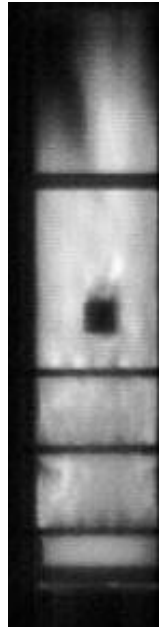

(e)

Figure 8. Sequence of experimental images to show flame structure at different times after ignition (a) 4.5, (b) 6, (c) 10, (d) 10.5 and (e) $11.0 \mathrm{~ms}$. 


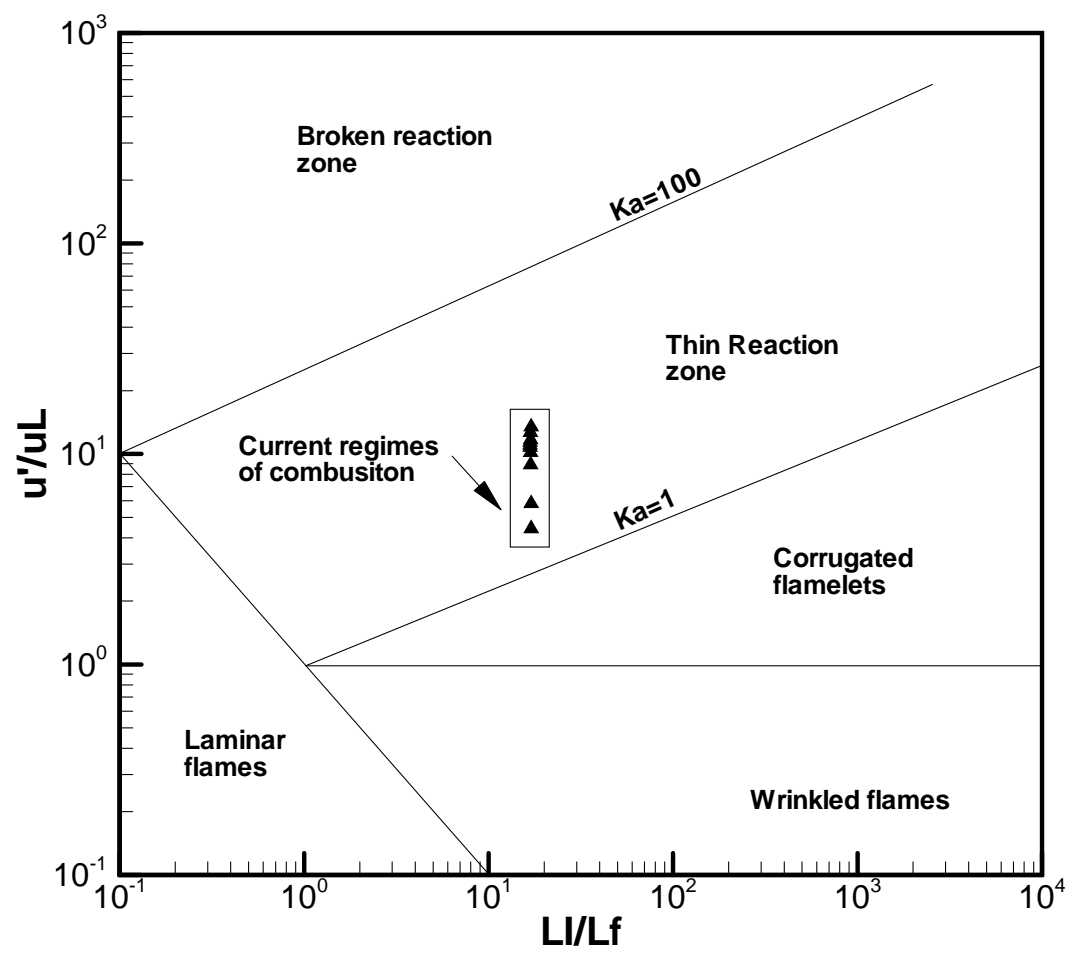

Figure 9a

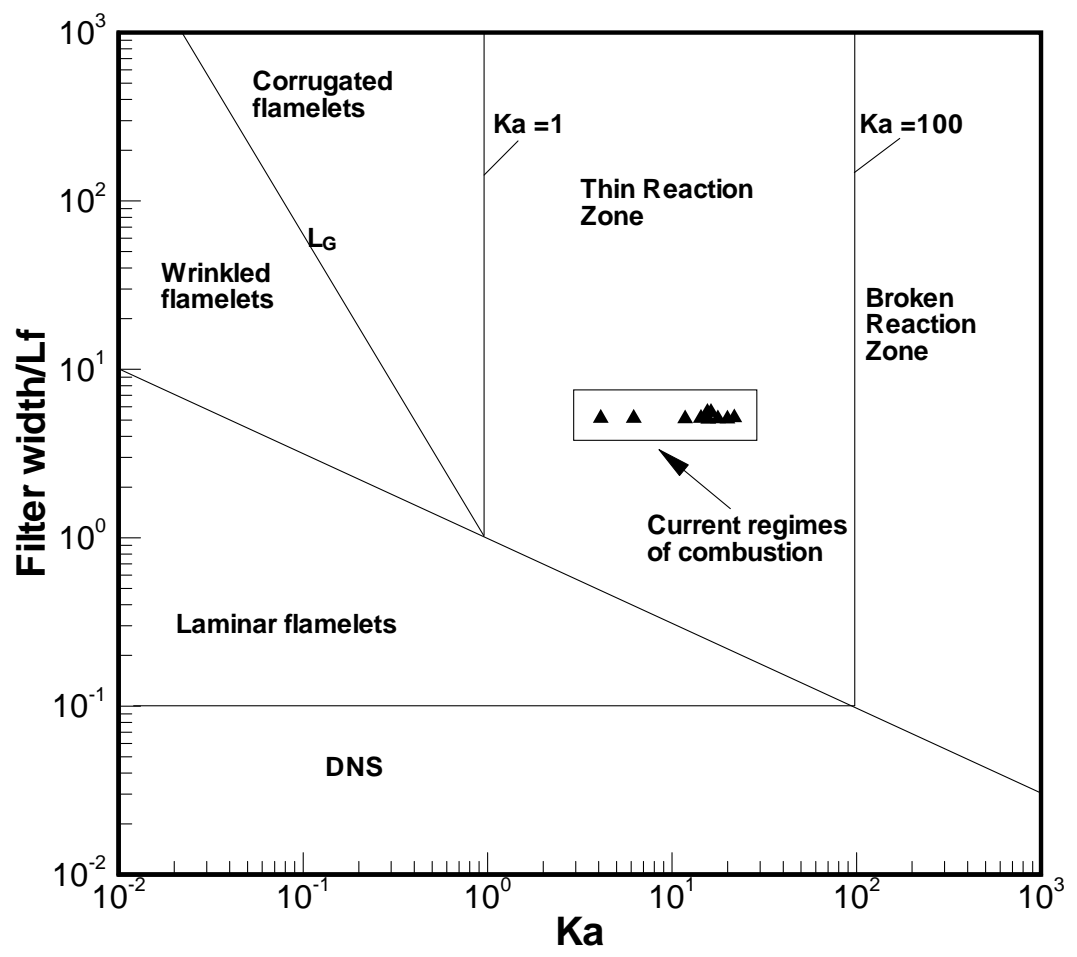

Figure 9b

Figure 9. Estimates from case $\mathrm{C}$ of the LES simulations presented in Table 3 are fitted in to the regimes of combustions (a) Turbulent premixed combustion reported by Peters [9] (b) LES turbulent premixed combustion reported by Pitsch \& De Lageneste [11]. 


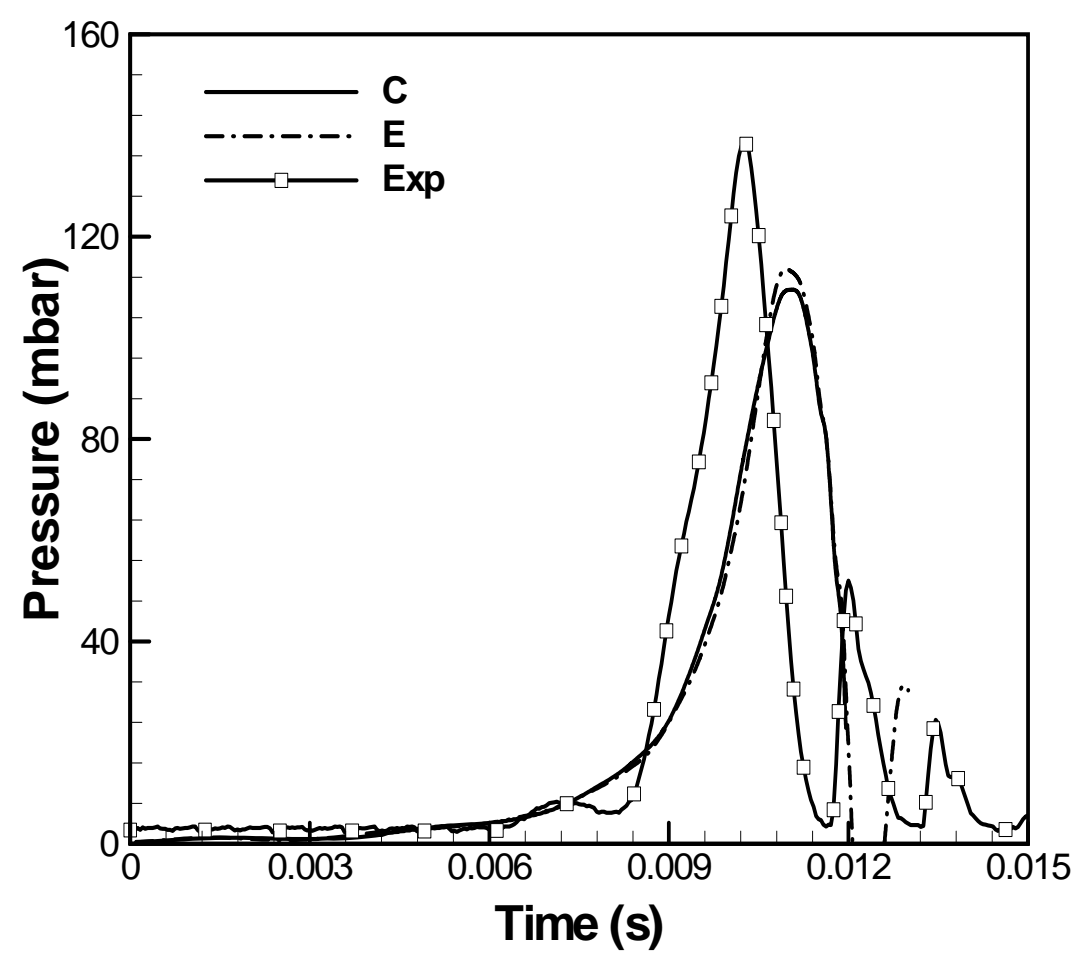

Figure 10a

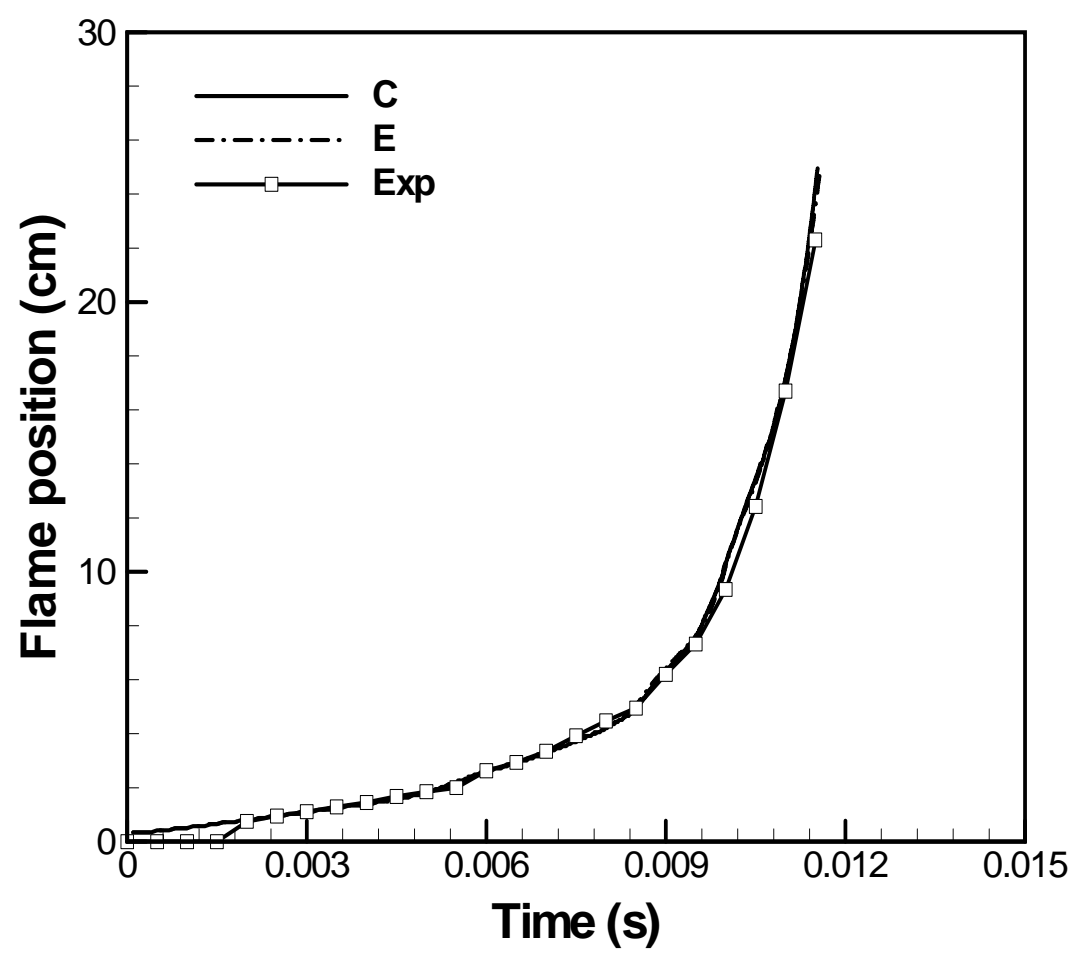

Figure 10b

Figure 10. Comparisons of LES simulations $\mathrm{C}$ and $\mathrm{E}$ with experimental measurements (a) Histories of overpressure (b) Flame position vs. time. 


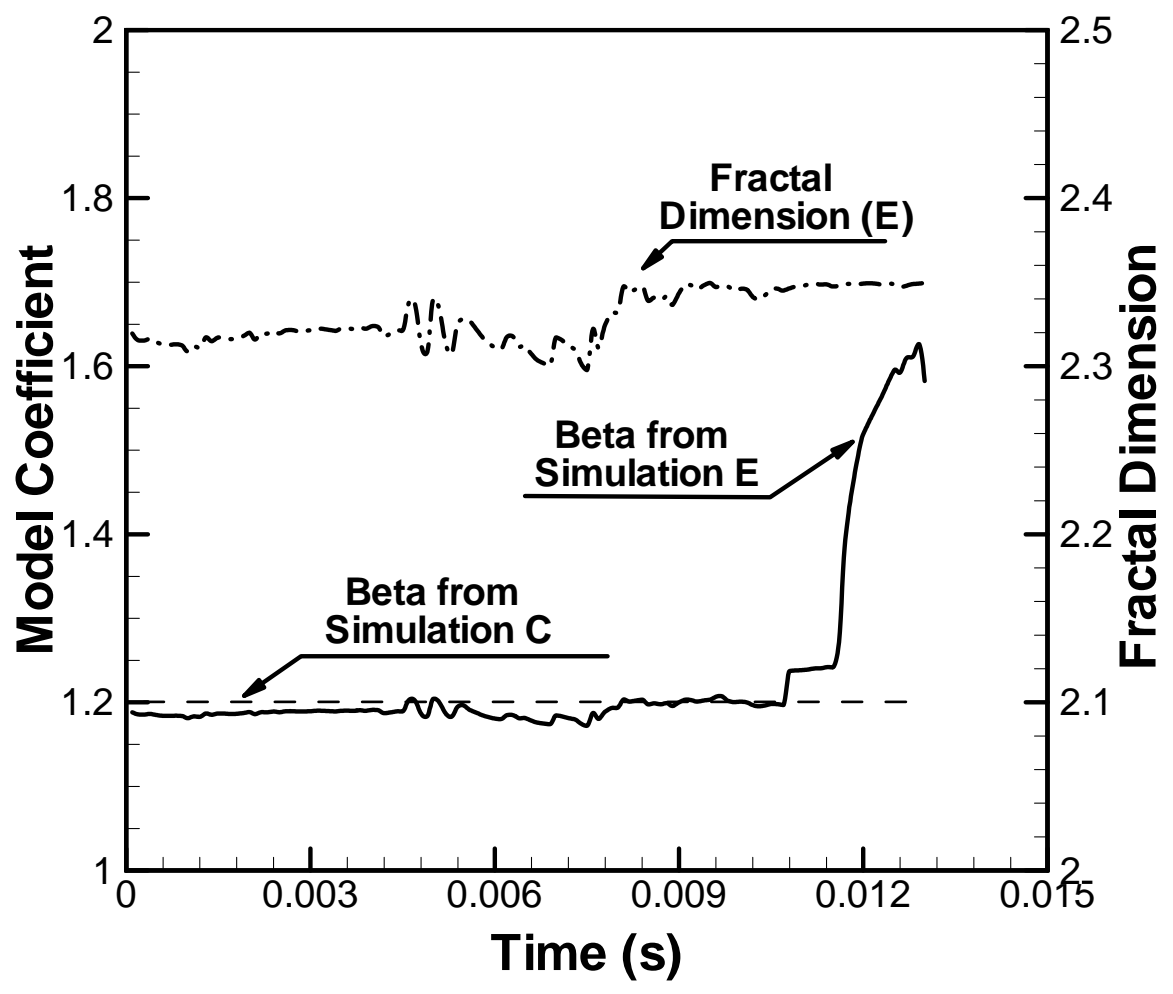

Figure 11. Time series of the model coefficient and fractal dimension using a sub-model in case $\mathrm{E}$ at the leading edge of the propagating flame. The dashed line is representing the constant model coefficient in case $\mathrm{C}$. 